Illinois State University

ISU ReD: Research and eData

Theses and Dissertations

$2-22-2020$

\title{
Mediation Among Maladaptive Perfectionism, Maladaptive Emotion Regulation, and Distress
}

Jennifer L. Woodrum

Illinois State University, woodrumjen@gmail.com

Follow this and additional works at: https://ir.library.illinoisstate.edu/etd

Part of the Clinical Psychology Commons

\section{Recommended Citation}

Woodrum, Jennifer L., "Mediation Among Maladaptive Perfectionism, Maladaptive Emotion Regulation, and Distress" (2020). Theses and Dissertations. 1206.

https://ir.library.illinoisstate.edu/etd/1206

This Thesis is brought to you for free and open access by ISU ReD: Research and eData. It has been accepted for inclusion in Theses and Dissertations by an authorized administrator of ISU ReD: Research and eData. For more information, please contact ISUReD@ilstu.edu. 


\section{MEDIATION AMONG MALADAPTIVE PERFECTIONISM, MALADAPTIVE EMOTION REGULATION, AND DISTRESS}

\section{JENNIFER L. WOODRUM}

\section{Pages}

Perfectionism is a personality trait characterized by high standards for performance (Frost, Marten, Lahart, \& Rosenblate, 1990) and a striving for flawlessness (Flett \& Hewitt, 2002). Maladaptive perfectionism, which has to do with self-critical thoughts (Dunkley, Zuroff, \& Blankstein, 2003) and feelings of falling short of high standards (Slaney, Rice, \& Ashby, 2002), is often associated with maladaptive strategies of emotion regulation (Aldea \& Rice, 2006; Dunkley et al., 2003; Rudolph, Flett, \& Hewitt, 2007). Additionally, research has shown that general use of maladaptive emotion regulation strategies mediates the relation between maladaptive perfectionism and distress (Aldea \& Rice, 2006; Di Schiena, Luminet, Philippot, \& Douilliez, 2012; Macedo et al., 2015). To my knowledge, however, this mediation had not been studied when an individual experiences momentary distress and must actively regulate his or her emotions. This study was designed to validate the general mediation model and then determine if this model occurs between maladaptive perfectionism, momentary maladaptive emotion regulation, and momentary distress when an experience of failure is induced. I elicited distress to determine if the level of failure that one faced would moderate the pathway between maladaptive perfectionism and maladaptive emotion regulation in the mediation model. Results showed that when all three mediators were examined in a structural equation model, typical use of rumination mediated the relation between trait levels of maladaptive perfectionism and typical distress, but 
typical use of suppression and reappraisal did not. In the momentary distress model, moderated mediation was present for one's momentary use of rumination but not for suppression and reappraisal. More specifically, momentary rumination mediated the path between maladaptive perfectionism and momentary distress in the low-failure condition but not in the high-failure condition. This finding demonstrates that for individuals high in maladaptive perfectionism, situations that result in extremely high distress may result in one detaching from his or her goals to avoid feeling inferior. Limitations, future directions, and implications are discussed.

KEYWORDS: perfectionism; emotion; suppression; rumination; reappraisal; distress 
REGULATION, AND DISTRESS

JENNIFER L. WOODRUM

A Thesis Submitted in Partial Fulfillment of the Requirements for the Degree of

MASTER OF SCIENCE

Department of Psychology

ILLINOIS STATE UNIVERSITY 
(C2020 Jennifer L. Woodrum 
MEDIATION AMONG MALADAPTIVE PERFECTIONISM, MALADAPTIVE EMOTION

REGULATION, AND DISTRESS

JENNIFER L. WOODRUM

COMMITTEE MEMBERS:

Jeffrey H. Kahn, Chair

Suejung Han 


\section{ACKNOWLEDGMENTS}

I would like to acknowledge everyone who played a role in my academic accomplishments. First, I am grateful for my family members. I would like to thank my husband who has supported me throughout the completion of my Master's. I am also grateful to my parents who instilled in me a passion for learning and a strong work ethic.

Secondly, I would like to acknowledge my committee members who have provided me with guidance, insight, and support throughout my research process. I would like to express my deep gratitude to Dr. Jeffrey Kahn and Dr. Suejung Han for providing me with the opportunity to conduct this study and inspiring me with their own research. I would also like to thank the undergraduate research assistants who put in many hours to help with data collection for my study. Finally, I am grateful to the faculty of the Clinical-Counseling Psychology Master's Program for their endless support and encouragement.

J.L.W . 


\section{CONTENTS}

Page

ACKNOWLEDGMENTS

TABLES

FIGURES

CHAPTER I: INTRODUCTION 1

CHAPTER II: REVIEW OF THE LITERATURE 4

$\begin{array}{ll}\text { Perfectionism } & 4\end{array}$

Frost Multidimensional Perfectionism Scale $\quad 5$

Hewitt and Flett Multidimensional Perfectionism Scale $\quad 6$

$\begin{array}{ll}\text { Almost Perfect Scale } & 8\end{array}$

Integration of Perfectionism Scales $\quad 9$

$\begin{array}{ll}\text { Emotion Regulation } & 12\end{array}$

Emotion Regulation in the Modal Model 13

Perfectionism and Emotion Regulation 18

Adaptive Perfectionism and Emotion Regulation 18

Maladaptive Perfectionism and Emotion Regulation 20

Mediation Among Maladaptive Perfectionism, Maladaptive Emotion Regulation, and

$\begin{array}{ll}\text { Distress } & 25\end{array}$

$\begin{array}{ll}\text { The Present Study } & 29\end{array}$

$\begin{array}{ll}\text { Hypotheses } & 32\end{array}$

CHAPTER III: METHOD

$\begin{array}{ll}\text { Participants } & 35\end{array}$ 
Instruments

Almost Perfect Scale-Revised (APS-R)

Frost Multidimensional Perfectionism Scale (F-MPS)

Emotion Regulation Questionnaire (ERQ)

Ruminative Responses Scale (RRS)

Depression Anxiety Stress Scales (DASS)

Positive and Negative Affect Schedule (PANAS)

State Perfectionism

Momentary Emotion Regulation

Procedure

Data Analysis Plan

Structural Equation Model of Trait Measures

Moderated-Mediation of Momentary Distress

General Model

Momentary Distress Model

Limitations of the Present Study

Implications

REFERENCES

APPENDIX A: THORNDIKE INTELLIGENCE MEASURE - HIGH-FAILURE CONDITION 
APPENDIX B: THORNDIKE INTELLIGENCE MEASURE - LOW-FAILURE

$\begin{array}{ll}\text { CONDITION } & 95\end{array}$

$\begin{array}{ll}\text { APPENDIX C: INFORMED CONSENT } & 97\end{array}$

$\begin{array}{ll}\text { APPENDIX D: DEBRIEFING } & 100\end{array}$

APPENDIX E: STRATEGIES QUESTIONNAIRE 102

APPENDIX F: STATE PERFECTIONISM QUESTIONNAIRE 103 


\section{TABLES}

Table

Page

1. Means and Standard Deviations for Maladaptive Perfectionism, Emotional Regulation

Strategies, and Distress Measures

2. Means and Standard Deviations for Momentary Emotion Regulation, State

Perfectionism, and Distress Measures after Manipulation

3. Correlations among Scores on Perfectionism, Emotion Regulation, and Distress

Measures 


\section{FIGURES}

Figure $\quad$ Page

1. The moderated mediation model that was expected for this study. 34

2. The conceptual model of the mediation analysis of typical maladaptive perfectionism, emotion regulation, and distress.

3. Factor loadings and standardized regression coefficients in the model of the mediation analysis of typical maladaptive perfectionism, emotion regulation, and distress.

4. Conceptual diagram of the momentary distress model of moderated mediation.

5. Standardized regression coefficients for the moderated mediation model for rumination are shown.

6. Standardized regression coefficients for the moderated mediation model for suppression are shown.

7. Standardized regression coefficients for the moderated mediation model for reappraisal are shown. 


\section{CHAPTER I: INTRODUCTION}

Personality traits can have an impact on daily functioning, both positively and negatively. Perfectionism, for example, can affect many different aspects of well-being including mood states (Wei, Mallinckrodt, Russel, \& Abraham, 2004; Wu \& Wei, 2008), psychopathology (Hewitt \& Flett, 1991; Limburg, Watson, Hagger, \& Egan, 2017), emotion (Perrone-McGovern, Simon-Dack, Beduna, Williams, \& Esche, 2015; Rice, Suh, \& Davis, 2018), cognition (Besser, Flett, Guez, \& Hewitt, 2008), and life satisfaction (Smith, Saklofske, Yan, \& Sherry, 2017). Studying maladaptive perfectionism is a particularly important task because of the negative outcomes associated with this trait. Maladaptive perfectionism was found to predict symptoms of anxiety, depression, and stress (Bieling, Israeli, \& Antony, 2004), which is a cause for concern.

The relation between perfectionism and distress has been studied in a variety of ways. Maladaptive perfectionism has been associated with distress in adolescents as a predictor of depression, anxiety, and self-harm (O’Connor, Rasmussen, \& Hawton, 2010). Among medical students, maladaptive perfectionism was highly correlated with distress, which included feelings of depression, hopelessness, and suicidal ideation, as well as neuroticism (Enns, Cox, Sareen, \& Freeman, 2001). Different traits of perfectionists have been found to be associated with a variety of experiences of feeling distressed, such as symptoms of depression and anxiety (Dunkley, Blankstein, Halsall, Williams, \& Winkworth, 2000). For example, perfectionism associated with critical self-evaluation was related to distress. This relation was mediated by hassles, avoidant coping, and perceived social support, meaning that these three factors could statistically explain why perfectionism and distress were related. Other mediators, or explanatory variables, in the relation between perfectionism and distress include repetitive negative thinking (Macedo et al., 
2015), self-criticism (James, Verplanken, \& Rimes, 2015), and maladaptive coping styles (Park, Heppner, \& Lee, 2010).

Another mediator of the relation between perfectionism and distress is emotion regulation, which was a variable of focus in the present study. Maladaptive perfectionism can be a predictor of both emotion dysregulation and distress, with emotion regulation acting as a mediator (Aldea \& Rice, 2006). For example, catastrophizing and rumination (Macedo et al., 2017; O’Connor, O’Connor, \& Marshall, 2007) have been found to mediate the relation between perfectionism and psychological distress, which included depression, anxiety, and hostility. These findings imply that perfectionism may be associated with difficulty working through emotions. Distressing symptoms of depression and anxiety may result from an excessive focus on negative emotions, which could have to do with the self-critical cognitions and disappointment related to perfectionism.

The relations between these variables are important to study because of the negative outcomes described above including symptoms of depression, anxiety, and distress. Symptoms of depression are often associated with negative outcomes including functional impairment, physical pain, and suicide attempts (American Psychiatric Association [APA], 2013). Symptoms of anxiety may impair productivity and lead to significant impairment and disability. These experiences may be even more difficult for perfectionists to cope with due to their high personal standards (Shafran \& Mansell, 2001).

These negative outcomes of perfectionism often require treatment. However, research has shown that therapeutic treatment of perfectionists can be difficult and complex (Blatt \& Zuroff, 2002). These treatment difficulties are likely because perfectionism has been shown to be related to impaired social support, a poorer therapeutic alliance, and hindered progress near the 
termination of therapy. Even more, some psychopathologies have been found to be associated with perfectionism including eating disorders (Goldner, Cockell, \& Srikameswaran, 2002) and obsessive-compulsive disorder (Rasmussen \& Tsuang, 1986). Understanding the outcomes of perfectionism can influence the treatment of these psychopathologies. In addition, knowing that the use of maladaptive emotion regulation strategies by perfectionists influences the negative outcomes they experience means that intervention could be targeted towards emotion regulation.

Whereas these variables have been studied in various populations including with coaches (Hill \& Davis, 2014), adolescents (O’Connor et al., 2010), and students (Enns et al., 2001), this relation has not been studied in the context of a specific, emotional event to my knowledge. Research has shown that emotion regulation mediates the relation between perfectionism and distress (Aldea \& Rice, 2006; O’Connor et al., 2007). At this time, it appears that in all of the studies that support this finding, typical use of emotion regulation and distress is measured rather than the in-the-moment experiences of emotion regulation and distress following a stressor.

The purpose of the present study was to explore the intricacies of the known relations among maladaptive perfectionism, emotion regulation, and distress in greater depth. More specifically, this study examined how these relations appear in the moment in response to a stressor. I investigated whether the relations among these three variables would be stronger when a high-level failure is experienced (i.e., momentary distress is experienced) compared to a situation in which a low-level failure is experienced. Understanding the impact of these different levels of failure would help us gain a greater understanding of maladaptive perfectionism, its negative outcomes, and how interventions should be targeted to decrease distress among perfectionists. 


\section{CHAPTER II: REVIEW OF THE LITERATURE}

\section{Perfectionism}

Perfectionism has been represented as both a functionally adaptive trait and an unhealthy, neurotic condition (Hamachek, 1978; Terry-Short, Owens, Slade, \& Dewey, 1995). Many definitions of perfectionism emphasize the setting of excessively high standards for performance, as well as overly critical self-evaluations (Frost, Marten, Lahart, \& Rosenblate, 1990). Whereas high standards could lead to success in some individuals, aspects of perfectionism can also manifest in destructive ways. Flett and Hewitt (2002) defined perfectionism as the striving for flawlessness, explaining that extreme perfectionists want to be perfect in all aspects of their lives. The DSM-5 includes rigid perfectionism as a secondary trait domain of personality disorders, defining it as a rigid insistence on everything being flawless, perfect, and without errors or faults, including one's own performance and the performance of others (APA, 2013).

Perfectionism can be conceptualized as both a trait and a type depending on the setting. Traits of perfectionism are evaluated on a scale or a continuum. For example, the multidimensional scales of perfectionism that will be described below consider different traits of perfectionism through subscales. These scales can determine that an individual has high levels of doubts about their actions or concerns over their mistakes, both of which are examples of traits of perfectionism.

On the other hand, individuals can be categorized into different types based on their traits of perfectionism. Many researchers attempting to define perfectionism have determined that there are multiple types of perfectionists based on the specifics of how the traits emerge in different individuals. For example, some researchers group together traits to determine the maladaptive and adaptive types of perfectionists. Maladaptive perfectionists as a type can be 
defined as those who score high on subscales that are considered to be maladaptive traits of perfectionism. Similarly, adaptive perfectionists are defined based on high scores on subscales that are considered to be more adaptive traits of perfectionism. Examples of these types will be described throughout the explanations of different scales below.

An early conceptualization of perfectionism by Hamachek (1978) distinguished between two types of perfectionists: normal perfectionists and neurotic perfectionists. Whereas normal perfectionists pay attention to their strengths, focus on how to do things right, and feel enhanced self-esteem at success, neurotic perfectionists cannot feel satisfied by their work and are motivated by a fear of failure. Hamachek described the behavioral symptoms of perfectionism as depression, a nagging "I should" feeling, face-saving behavior, shyness and procrastination, selfdeprecation, and feelings of shame and guilt. Whereas these symptoms can describe both normal and neurotic perfectionism, the neurotic expression of perfectionism involves greater intensity and duration of the symptoms.

Considering the complexity of the concept, many psychologists have turned to multidimensional scales to determine the different traits of perfectionism (Sironic \& Reeve, 2015). Hewitt and Flett (1991), for example, argued that perfectionism has both personal and social components. Other studies have found prominent factors of high standards, order, and the discrepancy between actions and expectations (Slaney, Rice, Mobley, Trippi, \& Ashby, 2001). To gain a comprehensive understanding of the current conceptualizations of perfectionism, I will examine its most common and refined methods of evaluation.

\section{Frost Multidimensional Perfectionism Scale}

Frost and colleagues (1990) developed the first multidimensional scale for analyzing perfectionism, which consists of six dimensions. These dimensions can be considered to be 
different traits of perfectionism. Concern over Mistakes includes negative reactions to mistakes, considering mistakes to be failures, and fearing the loss of respect after failure. Personal Standards is the setting of very high standards that hold excessive importance for self-evaluation. Parental Expectation is the belief that parents set very high goals for the individual, whereas Parental Criticism considers the overly critical evaluation of these goals. Doubts about Actions is the tendency to feel that one's work is not completed to a satisfying standard. Finally, Organization involves an emphasis on order. These six factors are measured by the 35 items of the Frost Multidimensional Perfectionism Scale (F-MPS). The scores from the scale show high internal consistency and reliability, as well as high correlations with the major components of other perfectionism scales. The subscales of the F-MPS can also be used to determine additional traits of perfectionism, which will be explained later on.

\section{Hewitt and Flett Multidimensional Perfectionism Scale}

Soon after, Hewitt and Flett (1991) presented a second multidimensional scale of perfectionism that differentiated to whom the perfectionistic behavior is directed and attributed: self-oriented, other-oriented, and socially prescribed. Sironic and Reeve (2015) described the differentiation between these traits of perfectionism as being either internally or externally motivated. Self-oriented perfectionism includes self-directed perfectionistic behaviors such as setting exacting standards for oneself, stringently evaluating and censuring one's behavior, and striving to avoid failure (Hewitt \& Flett, 1991). Self-oriented perfectionism has been found to have some negative outcomes, including self-criticism, self-blame, and clinical levels of psychopathologies. Other-oriented perfectionism involves many of these same cognitive processes, but the focus is on others rather than the self. These cognitive processes include placing importance on others being perfect, having unrealistic standards for others, and 
stringently evaluating the performance of others. Whereas other-oriented perfectionism was related to some personality disorder symptoms, it was not significantly related to many negative outcomes.

Socially prescribed perfectionism, on the other hand, involves people's beliefs that others have unrealistic standards for them, evaluate them stringently, and exert pressure on them to be perfect (Hewitt \& Flett, 1991). Socially prescribed perfectionism is considered the most maladaptive form of perfectionism in this conceptualization due to its relation to negative outcomes including self-criticism, self-blame, fear of negative evaluations, and negative affect. Socially prescribed perfectionism was more strongly associated with negative personality patterns and clinical symptoms than self-oriented perfectionism was. Self-oriented perfectionism had the next strongest association with distress-related outcomes.

These three traits of perfectionism are measured by the Hewett and Flett Multidimensional Perfectionism Scale (HF-MPS), which contains 45 items (Hewitt \& Flett, 1991). Self-oriented, other-oriented, and socially prescribed perfectionism are each measured by 15 items. The scores from the HF-MPS have been found to have validity and internal consistency, demonstrating distinct subscales that measure different concepts. When comparing the F-MPS with the HF-MPS, Frost, Heimberg, Holt, Mattia and Neubauer (1993) found substantial overlap between the Total Perfectionism score from the F-MPS, which is made up of all of the subscales of the F-MPS except for the Organization subscale, and the Self-Oriented and Socially Prescribed Perfectionism subscales in the HF-MPS. Multiple subscales were correlated between the two multidimensional scales, showing potential for integration. 


\section{Almost Perfect Scale}

A third conceptualization of perfectionism was formulated by Slaney et al. (2001) as a strictly intrapersonal measure, unlike the F-MPS and the HF-MPS (Sironic \& Reeve, 2015). The three traits of perfectionism of the Almost Perfect Scale-Revised (APS-R) include High Standards, Order, and Discrepancy (Slaney et al., 2001). These were determined by obtaining definitions and descriptions of participants who identified as perfectionists. High Standards involves a personal expectation for performance, whereas Order is a desire for neatness and organization. Finally, Discrepancy appears as distress associated with the perceived discrepancy between the desired standards and the individual's performance. In a later text, Slaney, Rice, and Ashby (2002) described Discrepancy as the perception that one consistently fails to meet the high standards one sets for oneself. Seeing the significant impact Discrepancy has on maladaptive manifestations of perfectionism, Flett, Mara, Hewitt, Sirois, and Molnar (2016) evaluated the construct further to find two distinguishable factors of Discrepancy. Whereas one was found to be pure discrepancy, as defined above, a second was dissatisfaction, which refers to feelings of dissatisfaction after falling short.

These traits of perfectionism can be used to determine different types of perfectionists. Slaney et al. (2001) concluded that these subscales were valid measures of positive and negative aspects of perfectionism, with Discrepancy greatly contributing to the negative manifestations. Further supporting this notion, a cluster analysis of the APS-R determined that using the Discrepancy and High Standards subscales, three new types of perfectionism could be found (Rice \& Ashby, 2007). Adaptive perfectionists had high levels of High Standards and low levels of Discrepancy, whereas nonperfectionists had low levels of both. The third category of 
maladaptive perfectionists included high levels of High Standards, as well as high levels of Discrepancy.

\section{Integration of Perfectionism Scales}

Some researchers have used factor analysis to find higher-order factors and correlations between these multidimensional scales (Frost et al., 1993; Sironic \& Reeve, 2015). Many of these evaluations focus on what type of effect the traits of perfectionism have on an individual. For example, Terry-Short et al. (1995) compared multiple perfectionism scales to make a distinction between positive perfectionism and negative perfectionism. The type of reinforcement for a behavior, positive or negative, resulted in either positive or negative perfectionism, respectively. In other words, positive perfectionism resulted when someone experienced positive reinforcement (e.g., rewards, praise, approval) for their perfectionistic behaviors, whereas negative perfectionism resulted when someone experienced negative reinforcement (i.e., avoiding negative consequences) for their perfectionistic behaviors. The researchers also noted that the dimension of positive perfectionism could be further differentiated based on whether the positive reinforcement comes from the self or from others, mirroring the self-oriented and socially prescribed subscales of the HF-MPS. Positive perfectionism could originate from personal positive reinforcement, as in self-oriented perfectionism, or positive reinforcement from others, as in socially prescribed perfectionism. Whereas this distinction could be made for positive perfectionism, there was no difference in negative perfectionism based on how the negative reinforcement was prescribed (personally or socially).

Sironic and Reeve (2015) found two higher-order factors through factor analysis of the FMPS and HF-MPS. These two factors can be viewed as traits of perfectionism. The first new trait, Positive Striving, included high scores on the Self-Oriented and Other-Oriented subscales 
of the HF-MPS and high scores on the Personal Standards and Organization subscales of the FMPS. Positive striving perfectionism was related to high levels of positive affect. The second trait, Evaluative Concerns, included high scores on the Socially Prescribed subscale of the HFMPS and high scores on the Concern over Mistakes, Doubts about Actions, Parental Expectations, and Parental Criticism subscales of the F-MPS. The evaluative concerns trait of perfectionism was related to negative affect, depression, and low self-esteem, making it a maladaptive trait.

Many studies that use the F-MPS and the HF-MPS define another maladaptive trait of perfectionism — self-critical perfectionism — based on multiple components of both multidimensional scales. In a study by Dunkley, Zuroff, and Blankstein (2003), self-critical perfectionism was comprised of high levels of socially prescribed perfectionism from the HFMPS, high scores on the Concern over Mistakes and Doubts about Actions subscales of the FMPS, and high levels of self-criticism from the Depressive Experiences Questionnaire (DEQ). Another study by Aldea and Rice (2006) defined self-critical perfectionism using the first three subscales described above but not the self-criticism factor from the DEQ.

Finally, Sironic and Reeve (2015) found four higher-order perfectionism dimensions from an analysis of all three multidimensional scales described above. They used these dimensions to develop six subgroups, or types of perfectionists, to provide a broader understanding of perfectionistic manifestations. The two maladaptive subgroups were Externally Motivated Maladaptive Perfectionists, in which high standards were prescribed by others, and Mixed Maladaptive Perfectionists, in which there was a mixture of personal high standards, others-prescribed high standards, and distress when expectations are not met. The analysis also found a subgroup of Adaptive Perfectionists. These individuals did not experience external 
pressures, but rather they had personal high standards while also accepting results that were not perfect. Three subgroups of Nonperfectionists were found with their differentiation determined by values of order and levels of standards.

In summary, factor analyses of the multidimensional scales have revealed higher-order dimensions of perfectionism based on combinations of different subscales. Whereas the multidimensional scales of perfectionism have been highly utilized to determine traits of perfectionism, Shafran, Cooper, and Fairburn (2002) argued that these scales do not measure the construct of perfectionism itself but rather relevant variables. They stated that these variables describe processes that arise from perfectionism and contribute to clinically relevant perfectionism including fear of failure, dichotomous thinking, the need for self-control, evaluation of performance, and failure to meet standards.

Perfectionism can be viewed as both an adaptive and beneficial trait, as well as a maladaptive hindrance to functioning. Multidimensional scales describe different components of perfectionism, whereas other conceptualizations evaluate the effect these components have on individuals like normal versus neurotic (Hamachek, 1978), maladaptive versus adaptive (Slaney et al., 2001) and positive versus negative perfectionism (Terry-Short et al., 1995). As described above, some of these traits of perfectionism have been found to be related to negative outcomes. Many of these negative outcomes have to do with emotions such as fear of failure, negative affect, and low self-esteem. Due to the connection between perfectionism and emotion, it is important for us to understand what is currently known about emotion and how individuals regulate their emotions. 


\section{Emotion Regulation}

Emotion has been described and defined in many ways. Mayer and Geher (2004) described emotion as a feeling state that provides information about interpersonal experiences. Gross (1998) described emotion as complex, multidimensional, and both a biological and a social process. Cabanac (2002) explained that emotion contains the four dimensions of sensation and consciousness, which include the quality of the mental event, its intensity, its pleasure, and its duration.

An early discussion about emotion touches on whether the emotion is a mental experience or the bodily expression of the mental experience (James, 1884). Gross (1998) supported James' proposition, which is that emotion is the bodily changes that occur after the perception of the mental excitation. Gross described this as a response tendency that may or may not lead to a behavioral reaction. This definition of emotion is elaborated upon in the modal model of emotion, which I will use as a basis for my later exploration of emotion regulation (Gross \& Thompson, 2013).

The modal model of emotion presents a process of emotional experiences, containing four components that occur in a specific order (Gross \& Thompson, 2013). This model will be used later to describe the times at which emotion regulation may occur. Gross (2008) called the

first component of the modal model the situation. Situations, which may be internal and external, are stimuli that elicit an emotional response. The second component is attention, which means that emotions arise when an individual pays attention to the stimuli. The third component, appraisal, is defined by how the individual makes meaning of the situation. Finally, the fourth component of the modal model is the emotional response, which includes three components: the emotional experience, the behavioral response, and the physiological response. 


\section{Emotion Regulation in the Modal Model}

Gross (2008) built on the modal model of emotion and the earlier cited definition of emotion to explain the complex processes of emotion regulation, which refers to the processes by which we influence our emotional experiences (Gross, 1998). He explained that individuals regulate their emotions through processes used to change the emotional experience in a variety of ways. Individuals may alter the emotion itself, the temporal experience of the emotion, or the expression of the emotion. The process model of emotion regulation includes five types of emotion regulation strategies that occur at the different points of the modal model of emotion. First, I will discuss the five strategies, and then I will explore what is known about the effectiveness of them.

The first strategy, situation selection, occurs before the situation phase of the modal model (Gross, 1998). Situation selection consists of individuals avoiding or approaching specific situations based on what type of emotional reaction they expect the situation may elicit. Situation modification is the second strategy that occurs during the situation component of the modal model. This strategy consists of active steps that are taken to alter the situation directly, which will, in turn, change the resulting emotional experience. Situation modification is closely related to active coping strategies and problem-solving (Gross, 2008).

The third emotion regulation strategy is attentional deployment, which occurs during the attention component of the modal model (Gross \& Thompson, 2013). Attentional deployment has to do with an individual focusing their attention on different aspects of the situation. Three more specific strategies fall within this phase of the process model (Gross, 1998). Distraction is the first type of attentional deployment. Distraction consists of directing one's attention either away from the situation or towards aspects of the situation that do not elicit an emotional 
response. For example, an individual who is hearing bad news that would typically lead to sadness might use distraction by thinking about more positive stimuli. Concentration is the second type of attentional deployment, which involves directing focus and cognitive resources towards a different activity. Whereas distraction involves turning attention away from the situation or its emotional aspects, concentration involves the absorption of cognitive resources by a new activity, which may lead to an immersive, transcendent state called flow. Concentration may also involve directing focus towards a specific emotion to have greater control over it. Finally, the third type of attentional deployment, rumination, involves focusing one's attention on the feelings related to the emotional experience, as well as the consequences of the feelings. Rumination can be separated into two components: reflection and brooding (Treynor, Gonzalez, \& Nolen-Hoeksema, 2003). Whereas reflection involves cognitive problem-solving using thoughtful, inward investigations, brooding involves comparisons between an individual's current situation and an unachieved standard, similar to the Discrepancy component of perfectionism.

The fourth emotion regulation strategy is cognitive change, which occurs during the appraisal process of the modal model (Gross \& Thompson, 2013). Individuals may work to change their beliefs about their ability to manage the situation or change the mental processes that they use to modify the perception of the emotion-eliciting stimuli (Gross, 1998). Some examples of cognitive change are denial, isolation, intellectualization, cognitive reframing, and reappraisal. Whereas cognitive reframing is the process of reinterpreting an event to see more positive, alternative outcomes, reappraisal involves changing the emotional impact of a situation through cognitive alterations. 
The fifth and final emotion regulation strategy is response modulation, which occurs during the fourth component of the modal model (Gross, 1998). Response modulation is the process of influencing the physiological, experiential, or behavioral responses to a situation rather than the cognitions related to the emotions. An example of response modulation is expressive suppression, in which an individual attempts to inhibit their expressive behaviors related to the emotion (Gross, 2008).

Now that I have reviewed the components of emotion regulation and the known strategies, I will discuss what is known about the effectiveness of the strategies that are most relevant to the present study. The outcomes of reappraisal and suppression, for example, have been reviewed by Gross and John (2003). Reappraisal was found to be an effective emotion regulation strategy. There were positive outcomes related to reappraisal including coping through reinterpretation, increased positive emotion, closer relationships, and increased social sharing of emotions. Other benefits related to reappraisal included decreased levels of neuroticism, less negative emotion, less negative-emotion expression, and fewer depressive symptoms.

Suppression, on the other hand, was less effective and was related to more negative outcomes (Gross \& John, 2003). For example, suppression was related to self-reported inauthenticity and increased negative emotions. However, the increase of negative emotions may be due to the self-acknowledged inauthenticity of the individuals using suppression. In addition, suppression was related to maladaptive outcomes including decreased social sharing of emotion, decreased positive emotion, and decreased social support. Importantly, individuals using suppression experienced a greater number of depressive symptoms, decreased life satisfaction and self-esteem, and overall lower levels of well-being. 
Other research has confirmed and elaborated upon these findings, including a study by Dryman and Heimberg (2018). Reappraisal was not often used by individuals with major depressive disorder and social anxiety disorder, with the latter group believing that they were not capable of using cognitive reappraisal. Suppression was over-utilized by individuals with social anxiety disorder and individuals with trait depression. Also, suppression has been found to be related to worse treatment outcomes for individuals experiencing psychopathologies (Scherer, Boecker, Pawelzik, Gauggel, \& Forkmann, 2017). Individuals who utilized suppression were less likely to respond to therapy, possibly due to their difficulty discussing emotional experiences. These findings show that in general, the use of suppression has been found to be related to negative outcomes.

Whereas the studies described above have been correlational, our understanding of reappraisal and suppression can be further developed by an experimental study that explored both spontaneous and instructed use of two strategies (Ehring, Tuschen-Caffier, Schnülle, Fischer, \& Gross, 2010). The studies described above evaluated correlations between emotion regulation strategies and distress. Ehring and colleagues, on the other hand, instructed individuals to use either reappraisal or suppression while watching emotionally evocative film clips. Supporting previous research, they found that participants with histories of depression spontaneously used suppression more often than individuals who were never depressed. In contrast to what the researchers expected, all participants, including previously depressed individuals, benefited equally from using instructed reappraisal by displaying lower levels of negative mood. On the other hand, instructed suppression led to negative outcomes, such as greater levels of negative mood. 
Gross and John (2003) found that the use of suppression was positively correlated with the use of rumination. The researchers hypothesized that due to the lack of problem-solving associated with suppression, the use of this maladaptive emotion regulation strategy may lead to rumination, which is also associated with negative outcomes. The use of rumination was found to predict future symptoms of depression and anxiety (Just \& Alloy, 1997; Nolen-Hoeksema, 2000). Treynor and colleagues (2000) found that the reflection component of rumination had mixed results in its relation to depressive symptoms, but the brooding component of rumination was related to depressive symptoms both at the present time and longitudinally.

Given the strong association between rumination and negative emotional outcomes, it would be helpful to explore how rumination is considered a strategy that is used to regulate emotions. First, the act of ruminating, or directing one's thoughts to their emotional experience, is not always a negative form of coping, such as when one ruminates on positive emotional experiences (Martin \& Tesser, 1996). Even when thinking about negative emotions, Martin and Tesser argue that rumination can have a constructive intent. Some individuals may turn their attention to the emotional impact of a situation in an attempt to figure out ways that the situation can be changed or consider other ways the situation could have turned out. However, this strategy is associated with negative outcomes when individuals focus their attention on future difficulties through worry, past negative actions through regret, or the discrepancies between their performance and a goal.

In summary, I have described the well-supported findings that reappraisal is associated with positive outcomes, whereas suppression and rumination are associated with negative outcomes. Next, I will explore how these emotion regulation strategies are associated with different traits of perfectionism and the outcomes associated with their usage. 


\section{Perfectionism and Emotion Regulation}

Previous studies have shown a relation between perfectionism and emotion with findings that indicate an association between maladaptive perfectionism and negative affect (Ashby, Rice, \& Martin, 2006; Dunkley et al., 2003; Molnar, Reker, Culp, Sadava, \& DeCourville, 2006). It is important to gain an understanding of how maladaptive perfectionists may regulate the excessive amount of negative emotions they experience. Due to the significance of these relations, I will explore the specific types of emotion regulation that have been found to be associated with different traits of perfectionism. I will first summarize the relation between adaptive perfectionism and emotion regulation, followed by maladaptive perfectionism and emotion regulation. Finally, I will explore the relations among maladaptive perfectionism, maladaptive emotion regulation, and distress.

\section{Adaptive Perfectionism and Emotion Regulation}

Studies have found mixed results about adaptive perfectionism and emotion regulation strategies. In a study that focused on coping with distress, Dunkley et al. (2003) examined the relations between perfectionism, affect, and coping strategies using the F-MPS and the HF-MPS. The more adaptive trait of perfectionism, personal standards, had no significant relation with problem-focused coping strategies or distress. Similarly, a study by Berman, Nyland, and Burns (2007) found no correlations between adaptive perfectionism and the use of the suppression and rumination in any direction. Finally, in a longitudinal study, personal standards perfectionism was not associated with experiential avoidance at multiple time points over two years (Moroz \& Dunkley, 2019).

In a study that built on the research by Dunkley et al. (2003), Aldea and Rice (2006) found more significant results regarding emotion regulation. Also using the F-MPS and the HF- 
MPS, Aldea and Rice defined adaptive perfectionism by high scores on the Personal Standards subscale, whereas self-critical perfectionism was the maladaptive conceptualization of perfectionism, determined by high scores on the Concern over Mistakes, Doubts about Actions, and Socially Prescribed Perfectionism subscales. Adaptive perfectionism was associated with more effective emotion regulation strategies and lower levels of emotion dysregulation than selfcritical perfectionism. This association was a result that had not been found in the study by Berman et al. (2007) and Dunkley et al. (2003).

Many of these studies have focused on the F-MPS and the HF-MPS. Similar results were found in a study by Richardson, Rice, and Devine (2014) that used the APS-R, which includes the dimensions of Discrepancy, High Standards, and Order. In addition, the Emotion Regulation Questionnaire (ERQ), which assesses the use of reappraisal and suppression (Gross \& John, 2003), was used. Using the APS-R and the Anxiety subscale of the Sixteen Personality Factors Questionnaire, Richardson and colleagues (2014) categorized participants as maladaptive perfectionists, adaptive perfectionists, and nonperfectionists. Adaptive perfectionists, who had low scores on the Anxiety and Discrepancy subscales, used reappraisal more often than maladaptive perfectionists, who had high scores on these two subscales. Also using the APS-R, Rice, Vergara, and Aldea (2006) similarly found that the Discrepancy dimension was associated with less effective self-regulation strategies than the High Standards dimension.

On the other hand, Castro, Soares, Pereira, and Macedo (2017) found different results using the HF-MPS and the F-MPS. Surprisingly, personal standards and self-oriented perfectionism, which are considered to be more adaptive traits of perfectionism, were significantly, positively correlated with maladaptive emotion regulation strategies when distress was perceived. Self-oriented perfectionism was positively associated with self-blame and 
catastrophizing, whereas personal standards was positively associated with self-blame and blaming others. These mixed findings may have to do with the fact that self-oriented perfectionism and personal standards perfectionism have some negative features in addition to their adaptive features (Hewitt \& Flett, 1991; Stoeber, 2015).

Overall, adaptive perfectionism is inconsistently related to emotion regulation strategies. In some studies, no correlations were found. In others, adaptive perfectionism has been found to be related to adaptive emotion regulation strategies such as greater reappraisal (Richardson et al., 2014) and less emotional reactivity (Aldea \& Rice, 2006). Occasionally, adaptive perfection was related to maladaptive emotion regulation strategies such as self-blame and catastrophizing (Castro et al., 2017). Next, I will explore how maladaptive perfectionism is related to emotion regulation.

\section{Maladaptive Perfectionism and Emotion Regulation}

Unlike the inconsistent findings of adaptive perfectionism, maladaptive perfectionism has consistently been found to be strongly related to maladaptive emotion regulation strategies. The study by Dunkley et al. (2003) defined maladaptive, self-critical perfectionism by multiple indicators of the F-MPS and HF-MPS. Self-critical perfectionism was found to be associated with negative emotions when in the presence of stress, criticism, and self-doubt. Further, selfcritical perfectionism was significantly related to the use of dysfunctional avoidance when stressors were present. Dunkley and colleagues (2003) explained that the more specific strategies of disengagement and denial used by individuals high in self-critical perfectionism may further exacerbate their negative affect. Aldea and Rice (2006) also examined self-critical perfectionism and found that it predicted general emotion dysregulation. 
Some studies have examined emotion regulation strategies outlined by the Cognitive Emotion Regulation Questionnaire (CERQ), which evaluates nine different emotion regulation strategies that may arise when a person encounters a stressor (Garnefski, Kraaij, \& Spinhoven, 2001). Rudolph, Flett, and Hewitt (2007), for example, utilized the Perfectionism Cognitions Inventory (PCI), which evaluates frequencies of perfectionistic thoughts, and the CERQ. Rudolph and colleagues were specifically interested in the relation between self-oriented, otheroriented, and socially prescribed perfectionism, determined by the HF-MPS, and the emotion regulation strategies in the CERQ.

As the researchers predicted, there was a positive correlation between maladaptive, socially prescribed perfectionism and self-blame, rumination, and catastrophizing, all of which are maladaptive emotion regulation strategies (Rudolph et al., 2007). Further, socially prescribed perfectionism was negatively correlated with the use of healthy emotion regulation strategies like putting things into perspective and positive reappraisal. Self-oriented perfectionism, which has been found to be associated with some negative outcomes (Hewitt \& Flett, 1991), was associated only with self-blame (Rudolph et al., 2007). In general, individuals with high scores on the PCI experienced increased rumination and catastrophizing, as well as decreased positive appraisal, which is a more effective emotion regulation strategy.

Castro and colleagues (2017) also assessed emotion regulation strategies using the CERQ. In this study, maladaptive perfectionism was defined as the evaluative concerns trait of perfectionism, determined by the F-MPS and HF-MPS. Maladaptive perfectionism was associated with the problematic emotion regulation strategies of rumination, self-blame, blaming others, and catastrophizing, as the researchers expected. Maladaptive perfectionism was also negatively correlated with the more adaptive emotion regulation strategies of positive 
reappraisal, positive refocusing, and putting into perspective. This study also supported the finding of Rudolph et al. (2007) that self-oriented perfectionism was associated with self-blame (Castro et al., 2017).

Other research has supported the finding that rumination is related to maladaptive perfectionism. Malivoire and colleagues (2019) posited that individuals high in maladaptive perfectionism often turn their attention to negative information because of their high reactivity to perceived criticism. O'Connor et al., (2007) found the brooding component of rumination to be correlated with self-oriented and socially prescribed perfectionism. Macedo et al. (2017) also found rumination and catastrophizing to be significantly correlated with high levels of perfectionistic concerns determined by the HF-MPS and the F-MPS. Their study revealed high associations between these maladaptive emotion regulation strategies and perfectionistic cognitions. Finally, Flett, Madorsky, Hewitt, and Heisel (2002) found that rumination was correlated with high scores on the PCI for individuals with symptoms of depression.

Suppression, another maladaptive emotion regulation strategy, was also found to be associated with maladaptive perfectionism in a study by Bergman, Nyland, and Burns (2007). Using the Positive and Negative Perfectionism Scale, Bergman and colleagues supported the previously stated findings by showing that maladaptive perfectionism was associated with suppression, rumination, and demonstrating inflexibility when distressed. Supporting the findings by Bergman et al. (2007), Richardson and colleagues (2014) found that maladaptive perfectionism, determined by the APS-R, was associated with the use of suppression. Finally, Santanello and Gardner (2007) evaluated experiential avoidance in relation to maladaptive perfectionism determined by the F-MPS. Experiential avoidance, which is related to suppression (Hayes et al., 2004), was highly correlated with maladaptive perfectionism (Santanello \& 
Gardner, 2007). The relation between self-critical perfectionism and experiential avoidance was also confirmed in a longitudinal study, with self-critical perfectionism predicting increases in one's use of this maladaptive emotion regulation strategy over two years (Moroz \& Dunkley, 2019).

Whereas the studies reviewed above have been correlational, an experimental study by Van der Kaap-Deeder et al. (2016) assigned perfectionists to success and failure tasks to evaluate their use of rumination, avoidance, and acceptance. The researchers considered the evaluative concerns trait of perfectionism, determined by the F-MPS and the HF-MPS, to be a maladaptive trait of perfectionism. In the group in which a failure experience was induced, evaluative concerns perfectionism was associated with increased rumination and decreased acceptance regarding the failure 1 week later. Although the experience of failure did not significantly predict avoidance when perfectionism was present, Van der Kaap-Deeder and colleagues (2016) did find that avoidance was related to evaluative concerns perfectionism across both conditions.

Whereas many of the results are consistent regarding these findings, a study by PerroneMcGovern et al. (2015) had some varying findings. Similar to previous findings, maladaptive perfectionism, defined by high scores on the Discrepancy subscale of the APS-R, was significantly correlated with suppression. However, multiple-regression analyses revealed that maladaptive perfectionism did not contribute to variation in emotion dysregulation. In other words, maladaptive perfectionism did not predict emotion regulation strategy usage. The researchers explained that the difference between these findings and those of other studies could have been explained by the fact that the emotion regulation strategies were studied directly without the examination of anxiety and distress, which other studies typically include (PerroneMcGovern et al., 2015). 
Some researchers have attempted to explain why maladaptive perfectionists experience dysregulated emotions. Malivoire, Kuo, and Antony (2019) developed a model to demonstrate that multiple factors may influence the emotion dysregulation associated with maladaptive perfectionism. The authors explained that maladaptive perfectionists may first experience negative affect and decreased emotional awareness when they feel as though they are falling short of a standard. When this occurs, maladaptive perfectionists may feel guilt, shame, sadness, and anger due to a belief that they will be criticized and rejected by others (Malivoire, Kuo, \& Antony, 2019). These experiences can result in maladaptive perfectionists feeling unmotivated to change their negative emotions if they believe that their guilt or shame is justified because they did not meet their expected standards. The researchers explained that even if maladaptive perfectionists feel motivated to regulate their emotions, they are likely to use the maladaptive emotion regulation strategies described above either implicitly or explicitly due to their ineffective emotion regulation goals and their negative affect.

In summary, maladaptive perfectionism has been found to be associated with maladaptive emotion regulation strategies including disengagement, denial, self-blame, rumination, and catastrophizing. In addition, maladaptive perfectionism is related to decreased use of adaptive emotion regulation strategies, such as positive reappraisal and positive refocusing. Finally, maladaptive perfectionism is associated with the use of suppression, especially under distressing circumstances (Bergman et al., 2007).

These findings show that maladaptive perfectionism is related to maladaptive emotion regulation strategies, which are often related to distress-related outcomes, as described earlier. Due to this, it is important to look at all three of these variables and examine where the 
explanatory power of their relations lies. This leads me into a discussion of the mediating relations among perfectionism, emotion regulation, and distress.

Mediation Among Maladaptive Perfectionism, Maladaptive Emotion Regulation, and Distress

In addition to the correlation between maladaptive perfectionism and maladaptive emotion regulation, research has shown a strong relation between these two constructs and distress. For example, Richardson and colleagues (2014) explained that the problematic coping strategies of maladaptive perfectionists were associated with levels of chronic stress. In addition, Aldea and Rice (2006) found that self-critical perfectionism predicted both emotion dysregulation and emotional distress. More specifically, maladaptive emotion regulation has been found to mediate the relation between maladaptive perfectionism and distress. This mediation finding means that maladaptive emotion regulation is an underlying process that explains why maladaptive perfectionism is associated with distress. Because the present study builds on the relation between maladaptive perfectionism, emotion regulation, and distress, I will explore it in depth.

Some findings of the mediation relation are focused more closely on coping strategies, which have some similarities to emotion regulation strategies (Gross, Richards, \& John, 2006). For example, experiential avoidance, which has similarities to suppression (Hayes et al., 2004), has been found to mediate the relation between perfectionism and worry (Santanello \& Gardner, 2007). In a longitudinal study over two years, experiential avoidance also mediated the relation between self-critical perfectionism and symptoms of distress including depression and anxiety (Moroz \& Dunkley, 2019). In other studies, maladaptive coping mechanisms, including self- 
blame and avoidance, mediated the relation between self-critical perfectionism and both stress and negative affect (Dunkley \& Blankstein, 2000; Dunkley et al., 2003).

Focusing on emotion regulation strategies rather than coping strategies, Aldea and Rice (2006) supported the findings above by discovering that emotion dysregulation mediated the relation between perfectionism and distress. The researchers noted that the negative emotions related to maladaptive perfectionism may be worsened by overreactions to perceived failures, which could lead to the resulting distress. Further, the ineffective emotion regulation strategies used by individuals with high levels of maladaptive perfectionism may worsen their psychological distress and prevent them from moving on from these failures. Citing the work of Dunkley and colleagues (2003) regarding coping mechanisms, Aldea and Rice (2006) suggested that future research could examine emotion regulation and coping as mediators of the relation between perfectionism and distress, considering that coping was found to be only a partial mediator.

Rumination is one of the specific maladaptive emotion regulation strategies that has been found to mediate the relation between perfectionism and distress, specifically in the context of depression (Di Schiena, Luminet, Philippot, \& Douilliez, 2012). In this study, maladaptive perfectionism was defined by high scores on the Concern over Mistakes and Doubts about Actions subscales of the F-MPS. Rumination, a maladaptive emotion regulation strategy, mediated the relation between maladaptive perfectionism and depression. Similarly, O'Connor and colleagues (2007) found that the brooding component of rumination mediated the relation between perfectionism and both distress and depressive symptoms, such as hopelessness. This finding was consistently true for socially prescribed perfectionism, the most maladaptive form of 
perfectionism in this conceptualization, and self-oriented perfectionism, which also has some maladaptive components.

Macedo et al. (2017) found that rumination and catastrophizing had mediating roles between perfectionism and psychological distress, including depression and anxiety. Senra, Merino, and Ferreiro (2017) additionally found that brooding, reflection, and immature defenses were mediators between self-oriented perfectionism and depressive symptoms, as well as socially prescribed perfectionism and depressive symptoms. Whereas these maladaptive emotion regulation strategies explained the relation between self-oriented perfectionism and depression, socially prescribed perfectionism was directly associated with depressive symptoms without the mediator present. This finding further supports the maladaptive aspects of this trait of perfectionism.

Cognitive emotion regulation, which includes reappraisal and rumination, was found to mediate the association between perfectionism, determined by the HF-MPS and F-MPS, and affect (Castro et al., 2017). More specifically, maladaptive emotion regulation strategies mediated the relation between even adaptive traits of perfectionism and negative affect. The researchers suggested that maladaptive perfectionism might be related to experiences of distress because of the relation between maladaptive perfectionism and the use of ineffective emotion regulation strategies. More specifically, individuals with high levels of self-critical perfectionism may be more vulnerable to negative affect because of their increased use of rumination, selfblame, blaming others, and catastrophizing.

Overall, the relation between perfectionism and distress, mediated by maladaptive emotion regulation, has been found in multiple studies. The specifics of these findings vary based on the constructs being studied. Experiential avoidance mediated the relation between 
perfectionism and worry. Maladaptive coping mediated the relation between self-critical perfectionism and stress. Rumination, brooding, and catastrophizing mediated the relation between perfectionism, especially maladaptive perfectionism, and distress, which included depression and anxiety.

These findings emphasize the negative impact of problematic emotion regulation strategies related to perfectionism. We can understand why this relation is present through our understanding of perfectionism. By definition, maladaptive perfectionists are excessively selfcritical (Frost et al., 1990) and experience feelings of discrepancy between their high personal standards and their performance (Slaney et al., 2001). Due to these traits, maladaptive perfectionists might have a difficult time coping with stressors or results that fall even slightly below their standards (Dunkley et al., 2003). When facing a situation that may elicit negative emotions, the discrepancy component of maladaptive perfectionists may be activated, meaning that their feeling of falling short of their standards comes to the center of their awareness (Bieling et al., 2003).

One of the perfectionist's high standards for performance may include a desire to appear "put together," presenting positive emotions and maintaining emotional stability in the pursuit of being flawless. This may be an example of perfectionistic self-presentation, which represents one's desire to conceal their perceived shortcomings or imperfections (Hewitt et al., 2003). The desire for perfectionism could lead to a goal of not experiencing negative emotions at all if this would be considered unacceptable. When facing the situation stage of the process model, in which a negative emotion may be elicited, this discrepancy may be activated if perfectionists feel that by displaying negative emotions, they are falling short of a personal standard of being composed at all times. 
Whereas this reasoning could explain why maladaptive perfectionists experience emotion dysregulation during specific situations using Gross's modal model (1998), many of the studies have shown that maladaptive perfectionists experience maladaptive emotion regulation in general, rather than in specific situations. I speculate that maladaptive perfectionists exhibit typical use of rumination and suppression due to a similar explanation. If maladaptive perfectionists experience self-critical thoughts, fear failure, and feel like they are always falling short of their expectations, their cognitive resources may be focused on trying to measure up to their standards (Desnoyers \& Arpin-Cribbie, 2015). This excessive focus may leave few cognitive resources available for regulating emotions. When facing disappointment in their performance, both adaptive and maladaptive perfectionists may have a difficult time accepting difficult circumstances and seeing the positive aspects of a stressful situation because of their high personal standards (Rice, Vergara, \& Aldea, 2006). This would explain their decreased use of adaptive emotion regulation strategies, such as positive reappraisal, in a failure situation.

The mediating effect of maladaptive emotion regulation on the relation between maladaptive perfection and distress has been clearly supported through multiple correlational studies. Knowing that maladaptive emotion regulation strategies explain the relation between maladaptive perfectionism and distress in general, there is an opportunity to expand our understanding to emotion regulation and distress in the moment.

\section{The Present Study}

The present study built on the current understanding that perfectionism is related to distress with maladaptive emotion regulation as a mediator. This finding has been well-supported in many studies in which participants are asked about their perfectionism, emotion regulation, and distress in general. However, this study examined how this relation would manifest in the 
moment. The majority of these existing studies assess the typical use of emotion regulation and typical levels of distress. I investigated how these experiences vary in the presence of a distressing experience (i.e., failure) rather than in general. How does the mediation model differ when maladaptive perfectionists are exposed to an intense stressor, which was an induced highfailure experience, compared to a low-level stressor, which was an induced low-failure experience?

This research question is important for several reasons. First, having an understanding of this mediation relation in the present moment will have implications on our understanding of perfectionism, its potentially negative outcomes, and the emotional reactance of perfectionists. Generalized self-reports of the typical use of emotion regulation may not be representative of how individuals experience emotion in the moment due to respondent bias or a lack of selfawareness. Studying emotion regulation as the strategies used in the moment may lead to a more accurate report. To my knowledge, the experiential emotion regulation and resulting distress of perfectionists in response to a distressing situation has not been studied. As Dunkley (2018) posited, future studies on perfectionism should use experimental stimuli and assess the cognitive response that follows, which the present study did.

Second, it is important to examine the three emotion regulation strategies selected for this study. Reappraisal, an adaptive emotion regulation strategy, may be used less often by maladaptive perfectionists in response to a stressor due to the activation of their fear of failure. The maladaptive perfectionist's self-critical cognitions and disappointment at falling short of standards may keep them from being able to view alternative understandings of a negative encounter. In other words, their frustration at themselves with their perceived failure may be so strong that they are unable to consider other explanations or interpretations of a situation in 
which they fall short besides blaming themselves. Similarly, rumination should be considered due to the strong emotional reaction that perfectionists would likely experience when failing. Due to the importance they place on their high standards, maladaptive perfectionists may turn their attention towards their negative emotions, especially their disappointment of falling short. Finally, when maladaptive perfectionists find that they cannot control their negative thoughts about their emotional response or negative situation, they may resort to expressive suppression. In other words, they may push down their negative reaction during the response stage if they have a personal expectation of appearing composed. Further, they may try to cover up their emotions in an attempt to avoid thinking about the failure.

Finally, it is important to examine how the mediation relation might be moderated by a failure experience. We know that the relation between maladaptive perfectionism and maladaptive emotion regulation is present in general, but I questioned under what circumstances this relation is triggered. Are there any situations, such as an experience of failure, in which this mediation relation would exist in comparison to other situations in which it would not? Knowing what degrees of stress prompt this mediated relation would give us a deeper understanding of exactly what leads maladaptive perfectionists to experience negative outcomes. It is likely that, in comparison to individuals who experience a low level of failure, the experience of a perceived high level of failure would lead to greater emotion dysregulation and distress for individuals high in discrepancy. Although no studies to my knowledge have examined how individuals with perfectionism respond to different levels of stress, research has shown that perfectionism is associated with different responses in failure experiences compared to success experiences (Besser, Flett, \& Hewitt, 2004; Van der Kaap-Deeder et al., 2016). 


\section{Hypotheses}

The first aim of the present study was to investigate the previously verified findings of how maladaptive perfectionism is associated with distress and how emotion regulation acts as a mediator between these two entities. The present study conceptualized maladaptive perfectionism using three measures: high levels of discrepancy from the APS-R, concerns over mistakes from the F-MPS, and doubts about actions from the F-MPS. Discrepancy was selected for this study because of the well-established maladaptive properties of discrepancy, as well as the prevalence of the APS-R in perfectionism research since its development. The two measures from the F-MPS have also been shown to represent maladaptive traits. Including three measures of maladaptive perfectionism provided an additional means of verifying the findings of this study.

Based on previous findings, Hypothesis 1A was that trait levels of maladaptive perfectionism would be positively related to one's recent (i.e., past week) level of distress. Hypothesis $1 \mathrm{~B}$ was that this relation would be mediated by the typical use of maladaptive emotion regulation strategies (that is, higher suppression, higher rumination, and lower reappraisal). More specifically, I hypothesized that trait maladaptive perfectionism would be positively associated with general maladaptive emotion regulation, which would be positively associated with the recent level of distress.

Further, this study investigated how the momentary use of emotion regulation would mediate perfectionism and experiential distress during specific emotional events. Because discrepancy was defined by the perceived disparity between performance and high personal standards, it seemed likely that individuals with high levels of discrepancy would feel distressed by a situation in which they fell short of standards through an experience of high-level failure. 
Because the measure of maladaptive perfectionism included concern over making mistakes, I also anticipated that an experience of high-level failure would be more distressing than a lowlevel failure. An individual who strives to perform at a certain standard would likely feel more distressed as the distance between their performance and their standard increases. I expected that a more distressing situation (high-failure) would be associated with a stronger negative reaction. Due to the research findings that maladaptive perfectionism is associated with the use of maladaptive emotion regulation strategies in response to a failure situation (Van der KaapDeeder et al., 2016), I expected that this relation would become stronger as the intensity of the failure became stronger.

Due to this conclusion, Hypothesis $2 \mathrm{~A}$ was that, after experiencing a high degree of failure in a task (the high-failure condition), the positive association between maladaptive perfectionism and momentary distress would be mediated by in-the-moment use of maladaptive emotion regulation strategies when controlling for one's typical distress. More specifically, I hypothesized maladaptive perfectionism would be positively associated with the spontaneous use of maladaptive emotion regulation strategies, which would be positively associated with momentary distress.

On the other hand, I did not expect mediation in the low-failure condition. Therefore, Hypothesis $2 \mathrm{~B}$ was that the indirect effect of maladaptive perfectionism on distress through maladaptive emotion regulation would be greater in the high-failure condition than in the lowfailure condition (see Figure 1), with levels of typical distress being controlled. Specifically, I predicted that the level of failure experienced would moderate the path between maladaptive perfectionism and maladaptive emotion regulation such that the path would be stronger in the 
high-failure condition than in the low-failure condition. I did not anticipate that the failure manipulation would moderate the path between maladaptive emotion regulation and distress.

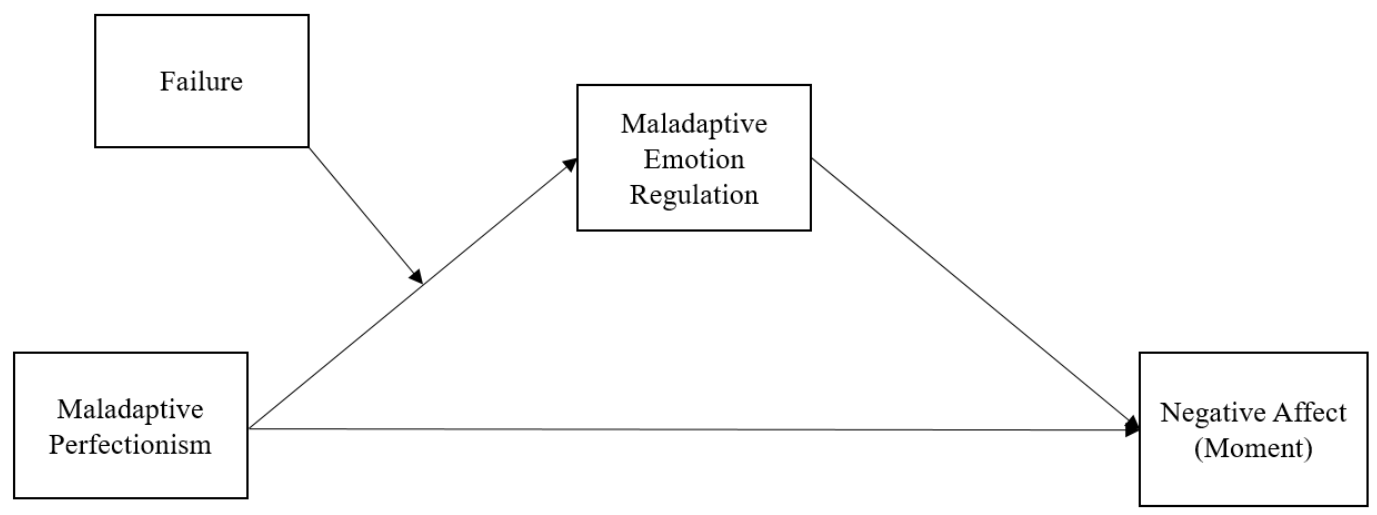

Figure 1. The moderated mediation model that was expected for this study. 


\section{CHAPTER III: METHOD}

\section{Participants}

Participants were students attending Illinois State University. Participants were recruited through the online sign-up system, SONA, run by the Illinois State University Department of Psychology. The minimum number of participants I had sought for this study was 100 students. Extra credit for psychology courses was offered as compensation for the students' time.

A total of 136 students (118 women, 86.8\%, and 18 men, 13.2\%) participated in this study. The participants' ages ranged from 18 to $35(M=19.53, S D=1.95)$. The majority of students identified as Caucasian/European American (66.9\%, 91 students), whereas 17 identified as Latino/Latina (12.5\%), and 16 identified as African-American (11.8\%). The remaining students identified as Asian-American (2.9\%, 4 students), Middle Eastern (2.2\%, 3 students), biracial or multiracial (2.2\%, 3 students), and other race or ethnicity (1.5\%, 2 students).

\section{Manipulation}

The failure experience was induced through a fabricated test of intelligence modeled after similar measures by Feather (1966), Lo and Abbott (2013), and Greenberg and Pyszczynski (1986). The intelligence measure, which was called the Thorndike Intelligence Measure, included 20 anagrams, which are words with the letters scrambled. Participants were instructed to rearrange each group of letters to create a meaningful English word. Participants were told that this test has been shown to be an accurate measure of intelligence. Modeling after Greenberg and Pyszczynski (1986), participants had 10 min to solve the 20 anagrams. A countdown was displayed on the computer screen while they completed the task.

For low-failure participants, the test consisted of 14 easy five-letter anagrams and 6 medium-to-difficult anagrams. Out of 45 participants in a previous study, these easy anagrams 
were able to be solved by between 24 and 44 participants (Gilhooly \& Johnson, 1978). For highfailure participants, the test consisted of 4 unsolvable, 12 medium-to-difficult, and 4 easy fiveletter anagrams that were used in previous studies. Out of 45 participants, the easy anagrams were solved by between 32 and 41 participants, whereas the difficult anagrams were solved by between 2 and 14 participants (Gilhooly \& Johnson, 1978). The unsolvable anagrams were both created for this study and borrowed from previous studies (Aspinwall \& Richter, 1999; Cackowski \& Nasar, 2003). I conducted a pilot study to test the Thorndike Intelligence Measure with 14 college-age individuals. The difficult version resulted in a mean score of 7.56 , and the easy version had a mean score of 10.00. Feedback was gathered from the pilot-study participants to make alterations for the final version to ensure that the measure felt realistic and possible to complete.

After completing the task, participants completed a questionnaire to assess their perception of their performance on the anagram test. Modeling after Greenberg and Pyszczynski (1986), the participants answered the question, "How do you feel about your performance on the Thorndike Intelligence Measure?" They responded on a 7-point Likert-type scale from extremely displeased to extremely pleased. This question helped assess whether or not the participants perceived their results as a failure. The manipulation check done by Greenberg and Pyszczynski (1986) revealed that, in a similar study, failure participants believed that they solved significantly fewer anagrams than the success participants did. Additionally, failure participants felt much worse about their performance than success participants did. Lastly, participants were asked how much effort they put into the Thorndike Intelligence Measure, with answers on a 7-point Likerttype scale ranging from none at all to a great deal. 


\section{Instruments}

\section{Almost Perfect Scale-Revised (APS-R)}

The APS-R is a self-report inventory that assesses levels of perfectionism using three subscales: Standards, Order, and Discrepancy (Slaney et al., 2001). The questionnaire includes 23 items on a 7-point Likert-type scale with options that range from strongly disagree to strongly agree. Seven items make up the Standards subscale, which assesses the high personal standards and expectations that one sets for oneself. Four items assess Order, including orderliness, neatness, and organization. Finally, 12 items assess Discrepancy, which is the disparity between one's personal high standards and the perception of their performance and success in reaching those standards. In this study, participants completed all three subscales, but only the Discrepancy subscale was used for analyses as one of three indicators of maladaptive perfectionism. Support for validity was found as the subscales were significantly correlated with related measures of perfectionism (Slaney et al., 2001). Maladaptive perfectionists are typically defined by having high scores on the Standards and Discrepancy subscales, whereas adaptive perfectionists and non-perfectionists have low scores on Discrepancy (Flett et al., 2016). In the present study, Cronbach's coefficient alpha ranged from .82 to .91 for scores from the three subscales.

\section{Frost Multidimensional Perfectionism Scale (F-MPS)}

The F-MPS is a self-report assessment that measures intrapersonal and interpersonal aspects of perfectionism (Frost et al., 1990). All items in this measure use a 5-point Likert-type scale with responses ranging from strongly disagree to strongly agree. In this study, I administered three subscales from the F-MPS: Personal Standards, Concern over Mistakes, and Doubts about Actions. Only the latter two subscales were used for analyses. The nine items from 
the Concern over Mistakes subscale and the four items from the Doubts about Actions subscale were used to measure maladaptive perfectionism. The F-MPS demonstrated validity as it was correlated with other measures of perfectionism, including the Burns Perfectionism Scale and the Eating Disorder Inventory Perfectionism Scale (Frost et al., 1990). Factor analysis also revealed evidence for internal consistency. In the present study, the scores from the Personal Standards, Concern over Mistakes, and Doubts about Action had coefficient alphas of .75, .86, and .71 respectively.

\section{Emotion Regulation Questionnaire (ERQ)}

The ERQ is a self-report assessment of the typical use of two emotion regulation strategies: reappraisal and suppression (Gross \& John, 2003). Six questions ask about reappraisal by questioning how participants change and restructure their emotions. Four questions evaluate suppression by asking participants how they keep themselves from expressing their emotions. The items use a 7-point Likert-type scale with responses ranging from strongly disagree to strongly agree. Evidence for construct validity has been displayed as both subscales were significantly correlated in the expected direction with related measures (Gross \& John, 2003). The reappraisal subscale was negatively associated with neuroticism and positively associated with reinterpretation, displaying evidence for discriminant and convergent validity, respectively. The suppression subscale was negatively associated with extraversion and positively associated with inauthenticity, displaying evidence for discriminant and convergent validity, respectively. In the present study, alpha reliability values ranged from .79 for the scores from the suppression scale to .86 for the scores from the reappraisal scale. 


\section{Ruminative Responses Scale (RRS)}

The RRS is a self-report questionnaire that assesses how much an individual uses rumination while feeling depressed or down (Treynor et al., 2003). The items are on a 4-point Likert-type scale with responses ranging from almost never to almost always when asked how often they think or do certain things in general. The participants completed 10 items, which represents a shortened version of the RRS. Twelve items that Treynor and colleagues deemed similar to the Beck Depression Inventory were removed from the scale for the present study, leaving 10 items that solely represent rumination. In the 10 -item scale, 5 items assess the reflection component of rumination, and 5 items assess the brooding component of rumination. The test-retest reliability was .67 (Treynor et al., 2003). There has been evidence for acceptable construct validity, including discriminant validity, through correlations with depression, trait anxiety, and neuroticism (Roelofs, Muris, Huibers, Peeters, \& Arntz, 2006). In the present study, the alpha coefficients were .81 and .75 for the scores from the brooding and reflection subscales respectively.

\section{Depression Anxiety Stress Scales (DASS)}

The DASS-21 is a 21-item self-report assessment that measures depression, anxiety, and stress through three scales (Henry \& Crawford, 2005). Each scale contains 7 items regarding experiences of symptoms over the past week. Participants respond on a 4-point severity and frequency scale with responses ranging from did not apply to me at all to applied to me very much, or most of the time. There was evidence for good convergent and discriminant validity for the depression and anxiety subscales (Henry \& Crawford, 2005). The DASS Anxiety scale was highly correlated with the Beck Anxiety Inventory $(r=.81)$, whereas the DASS Depression scale was correlated with the Beck Depression Inventory $(r=.74)$. The DASS-21 subscales were also 
associated in the expected directions with the Hospital Anxiety and Depression Scale and the Personal Disturbance scale. Factor analysis showed acceptable internal structure for each of the three subscales (Henry \& Crawford, 2005). In the present study, scores from the Depression, Anxiety, and Stress subscales had alpha values of $.85, .85$, and .84 , respectively.

\section{Positive and Negative Affect Schedule (PANAS)}

The PANAS is a self-report questionnaire that examines positive affect and negative affect as two dimensions of mood (Watson, Clark, \& Tellegen, 1988). Twenty items are presented on a 5-point Likert-type scale with responses ranging from very slightly or not at all to extremely when the respondents are asked about the extent to which they felt certain emotions. The Positive Affect subscale consists of 10 items that represent a positive mood, including feeling excited, enthusiastic, and proud. The Negative Affect Subscale consists of 10 items that represent a negative mood including feeling distressed, afraid, and nervous.

Two forms of the PANAS were presented to participants depending on the temporal instructions provided. In the first form of the PANAS, participants were asked about their experiences of positive and negative affect over the past week. In the present study, the pastweek PANAS had an alpha value of .86 for scores from the Positive Affect scale and .81 for scores from the Negative Affect scale. The second form of the PANAS asked participants about their experiences of positive and negative affect in the moment. This form of the PANAS had an alpha value of .89 for scores from the Positive Affect scale and .90 for scores from the Negative Affect scale in the present study. Factor analysis was used to support the validity of the assessment for each of the time frames used in the directions in the PANAS (Watson et al., 1988). Convergent correlations were .95 and .94 for momentary and general time frames, 
respectively. Construct validity and item validity have also been shown to be high for both scales.

\section{State Perfectionism}

The measure of one's level of perfectionism in-the-moment was created for this study by adapting items from the APS-R and the F-MPS. This measure was designed to assess how one's perfectionistic tendencies manifested while completing the Thorndike Intelligence Measure. Two items were adapted from the APS-R to measure one's feelings of discrepancy in regards to the anagram task. Six items were adapted from the F-MPS to measure personal standards, concern over mistakes, and doubts about action, with two items being used for each category. Only the items that measured discrepancy, concern over mistakes, and doubts about action were used in analyses. Sample items include "I probably set higher goals for myself than most people on this task" and "I felt disappointed after completing this task because I knew I could have done better." The 8 new items follow a 7-point Likert-type scale with responses ranging from strongly disagree to strongly agree. Validity and reliability were presumed to be similar to the findings of the APS-R, the F-MPS, and the HF-MPS, as the items in this measure of state perfectionism were only slightly modified. In this study, alpha reliabilities of the scores from the personal standards, concern over mistakes, doubts about actions, and discrepancy subscales were .53, .70, .69 , and .71 , respectively.

\section{Momentary Emotion Regulation}

Ehring et al. (2010) developed a Strategies Questionnaire to assess the active, momentary use of suppression and reappraisal when experiencing failure. Modeling after this measure, the Strategies Questionnaire for this study consisted of 3 items adapted from the ERQ to assess emotion suppression during the anagram task (e.g., "When I was feeling negative emotions, I 
made sure not to express them" and "I controlled my emotions by keeping them inside"). Three questions assessed the use of reappraisal during the anagram task (e.g., "If I started to get upset or frustrated, I changed the way I thought about my experience" and "I made myself think about this task in a way that helped me stay calm"). The 6 new items mirrored the ERQ in that they follow a 7-point Likert-type scale with responses ranging from strongly disagree to strongly agree. Validity and reliability were presumed to be similar to the findings of the ERQ, as the items in the Strategies Questionnaire were only slightly modified. In the present study, alpha reliabilities for scores from the suppression and reappraisal subscales were .71 and .74, respectively.

Three questions were added to the Strategies Questionnaire to assess the active use of rumination during the anagram task. These three questions were adapted from the RRS (e.g., "While doing this task, I thought about my shortcomings, failures, faults, and mistakes" and "While doing this task, I thought about how upset I was with myself"). To maintain consistency with the other questions of the Strategies Questionnaire, the items were adapted to be on a 7point Likert-type scale with responses ranging from strongly disagree to strongly agree when asked about their experience of completing the Thorndike Intelligence Measure. Reliability and validity were presumed to be similar to the findings of the RRS, as the items adapted for the Strategies Questionnaire were only slightly modified. Cronbach's alpha for the scores from the rumination subscale of the Strategies Questionnaire was .76 in the present study.

Three filler questions were added to assess participants' responses that were unrelated to the three emotion regulation strategies listed above (e.g., "I really enjoyed doing this task" and “This task made me feel good about myself”). The Strategies Questionnaire was reviewed by three research psychologists from different universities with experience in the area of emotion 
regulation. All three research psychologists were able to appropriately identify the type of emotion regulation being measured by each questionnaire item.

\section{Procedure}

Participants came to a lab for the completion of the study. Upon arriving, participants provided informed consent and set aside their cell phones or other electronic devices to minimize distractions. Participants were directed to a set of questionnaires on the computer including the APS-R, the F-MPS, the ERQ, the RRS, the DASS, and the general form of the PANAS. Once the participants completed the instruments, they received instructions about the Thorndike Intelligence Measure, modeled after Feather (1966). The researcher read the following instructions:

The test that you are about to receive is a test of your intelligence. Please try to do your best as scores on this test have been shown to indicate fair and accurate representations of your intelligence level. The test consists of twenty anagrams, which are disarranged words. Your task is to rearrange each group of letters so that they make a meaningful English word. You will have ten minutes to complete the Thorndike Intelligence Measure. There will be a timer on the screen in front of you. Start when you are so instructed and stop at the stop signal. Do not turn over the page until you are told to do so.

The participants in the low-failure condition were told that the average number of correct answers for college students was 12.1, whereas participants in the high-failure condition were told that the average number of correct answers for college students was 14.2. Participants received the Thorndike Intelligence Measure and were told to begin. After 10 min, the researcher told the participant to stop. The researcher then scored the Thorndike Intelligence Measure and 
told the participant how many anagrams they solved correctly. The researcher reminded the participant of the (fabricated) average score among college students. The researcher then directed the participant to the next set of questionnaires on the computer.

Participants completed the manipulation check, followed by the Strategies Questionnaire to assess their momentary use of the emotion regulation strategies. Participants then completed the state measure of perfectionism, followed by the PANAS to assess their current levels of positive and negative affect. This form of the PANAS asked participants about their experiences and feelings in the moment. After all questionnaires were completed, the researcher debriefed the participants on the purpose of the study. The explanation for the deception was part of the debriefing. Care was taken to ensure that participants understood that they had not actually failed or done poorly.

\section{Data Analysis Plan}

Descriptive statistics for study variables were first analyzed. Bivariate correlations between perfectionism, emotion regulation, and distress were tested using Pearson correlation analyses. This correlation analysis was used to test Hypothesis 1A, which was that maladaptive perfectionism would be positively related to one's recent level of distress.

Hypothesis 1B was that this relation would be mediated by one's typical use of maladaptive emotion regulation strategies. I used structural equation modeling to test this hypothesis. I used the lavaan package in $\mathrm{R}$ to determine the effect of maladaptive perfectionism on distress through three emotion regulation strategies: rumination, suppression, and reappraisal.

Hypothesis 2A was that the positive association between maladaptive perfectionism and momentary distress would be mediated by one's in-the-moment use of maladaptive emotion regulation strategies in the high-failure condition when controlling for one's typical distress. 
Hypothesis 2B was that the indirect effect of maladaptive perfectionism on distress through maladaptive emotion regulation would be greater in the high-failure condition than the lowfailure condition, with levels of typical distress being controlled.

To examine these two hypotheses, I used the lavaan package in $\mathrm{R}$ to conduct a mediation analysis. I used the measures of typical perfectionism, momentary emotion regulation, and momentary distress in this analysis, controlling for levels of typical negative affect. I used the measure of typical perfectionism rather than state perfectionism due to a lack of correlations between the state perfectionism measure constructed for this study and previously validated measures of trait perfectionism. To simplify the models, I examined the three mediators one at a time in three separate models. To assess Hypothesis 2A, I examined the indirect effect of maladaptive perfectionism on momentary distress through momentary use of maladaptive emotion regulation strategies for the high-failure condition.

This analysis also assessed the extent to which each mediator was moderated by the condition of the participant, meaning whether they experienced high failure or low failure. Thus, I combined the moderation and mediation results by estimating the conditional indirect effects of perfectionism on distress through emotion regulation as a function of high-failure and low-failure situations, using the moderated mediation approach described by Hayes (2018). To assess Hypothesis 2B, I examined the significance of the interaction term in this model and the index of moderated mediation, which shows whether or not the moderator has a significant effect on the indirect effect. The criterion for statistical significance was $p<.05$. 


\section{CHAPTER IV: RESULTS}

Participants completed five questionnaires prior to the manipulation to measure their general levels of perfectionism (APS-R and F-MPS), their emotion regulation (ERQ and RRS), and their distress (DASS). Table 1 displays the means and standard deviations for the 11 subscales of these five measures that were used for data analysis for all participants. For 9 out of

the 11 subscales, there were no significant differences between the low-failure condition and the high-failure condition prior to the manipulation on these measures of perfectionism, emotion regulation, and distress.

However, unexpectedly, there were significant differences between the two groups on two of the measures: the Discrepancy subscale of the APS-R and the Suppression subscale of the ERQ. The high-failure group had an average discrepancy score of $43.83(S D=12.89)$, and the low-failure group had an average discrepancy score of $39.42(S D=11.99)$. Hence, the highfailure group had significantly higher levels of discrepancy than the low-failure group before the manipulation, $t(134)=-2.07, p=.04, d=.35$. The high-failure group had an average suppression score of $15.23(S D=5.62)$, whereas the low-failure group had an average suppression score of $13.37(S D=5.11)$. Thus, the high-failure group reported significantly greater suppression than the low-failure group before the manipulation, $t(134)=-2.02, p=.046, d=.35$. 
Table 1

Means and Standard Deviations for Maladaptive Perfectionism, Emotional Regulation Strategies, and Distress Measures

\begin{tabular}{lcc}
\hline Variable & \multicolumn{2}{c}{ Total } \\
\hline & 41.62 & $S D$ \\
\hline APS-R-Discrepancy & 21.85 & 12.60 \\
FMPS-Concerns & 10.73 & 6.89 \\
FMPS-Doubts & 31.33 & 3.61 \\
ERQ-Reappraisal & 14.30 & 6.08 \\
ERQ-Suppression & 11.31 & 5.43 \\
RRS-Brooding & 11.30 & 3.72 \\
DASS-Anxiety & 13.15 & 4.29 \\
DASS-Stress & 10.27 & 4.50 \\
DASS-Depression & 33.10 & 3.66 \\
PANAS-W-Positive Affect & 21.29 & 7.71 \\
PANAS-W-Negative Affect & & 6.57 \\
\hline
\end{tabular}

Note. APS-R = Almost Perfect Scale-Revised; FMPS = Frost Multidimensional Perfectionism Scale; PS = Personal Standards; ERQ = Emotion Regulation Questionnaire; RRS = Ruminative Responses Scale; DASS = Depression Anxiety Stress Scale; PANAS-W = Positive and Negative Affect Scale-Week; $N=136$. 
There were significant differences between the two groups after the manipulation on the questionnaires taken after the Thorndike Intelligence Measure was completed. Table 2 shows the means and standard deviations for the two groups on these measures, as well as the $t$ values and effect sizes. Participants in the high-failure condition scored higher on the momentary use of reappraisal, state levels of doubts about actions regarding the anagram task, and momentary negative affect. There were no significant differences between the groups on the six other subscales of the post-manipulation measures. Participants in the high-failure condition correctly completed significantly fewer anagrams on the Thorndike Intelligence Measure than the participants in the low-failure condition, $t(134)=6.10, p<.001, d=1.05$. Participants in the high-failure condition also felt significantly less satisfied with their performance than those in the low-failure condition, $t(134)=5.80, p<.001, d=.99$. There was no difference between the groups in how much effort the participants put towards the Thorndike Intelligence Measure, $t(134)=-0.59, p=.56, d=.10$. 
Table 2

Means and Standard Deviations for Momentary Emotion Regulation, State Perfectionism, and Distress Measures after Manipulation

\begin{tabular}{|c|c|c|c|c|c|c|}
\hline \multirow[t]{2}{*}{ Variable } & \multicolumn{2}{|c|}{ Low Failure } & \multicolumn{2}{|c|}{ High Failure } & \multirow[t]{2}{*}{$t$} & \multirow[t]{2}{*}{ Cohen's $d$} \\
\hline & $M$ & $S D$ & $M$ & $S D$ & & \\
\hline SQ Suppression & 13.91 & 4.30 & 14.75 & 3.95 & -1.20 & .20 \\
\hline SQ Reappraisal & 13.56 & 3.60 & 12.91 & 3.14 & 1.03 & .19 \\
\hline SQ Rumination & 9.59 & 4.47 & 12.00 & 4.46 & $-3.15 * *$ & .54 \\
\hline SP Discrepancy & 8.29 & 3.29 & 9.23 & 3.14 & -1.71 & .29 \\
\hline SP Concerns over Mistakes & 5.29 & 2.65 & 6.15 & 3.02 & -1.75 & .30 \\
\hline SP Doubts about Actions & 8.03 & 2.87 & 9.77 & 2.63 & $3.67 * * *$ & .63 \\
\hline PANAS-M-PA & 24.71 & 9.38 & 22.19 & 7.01 & 1.77 & .30 \\
\hline PANAS-M-NA & 16.20 & 6.60 & 18.69 & 5.73 & $-2.34 *$ & .40 \\
\hline Score on Thorndike & 9.76 & 3.40 & 6.65 & 2.48 & $6.10 * * *$ & 1.01 \\
\hline Performance Satisfaction & 3.71 & 1.78 & 2.21 & 1.18 & $5.80 * * *$ & .99 \\
\hline Effort on Thorndike & 3.72 & 0.73 & 3.79 & 0.72 & -0.59 & .10 \\
\hline
\end{tabular}

Note. SQ = Strategies Questionnaire; SP = State Perfectionism; PANAS-M = Positive and

Negative Affect Scale-Moment; PA = Positive Affect; NA = Negative Affect, $N=136$.

$* p<.05 . * * p<.01 . * * * p<.001$.

Correlations were computed to examine the relations among perfectionism, emotion regulation, and distress (see Table 3). There were positive correlations between the typical 
measures of maladaptive perfectionism, maladaptive emotion regulation, and distress (see box with thin solid lines). First, discrepancy was positively correlated with the other measures of maladaptive perfectionism: concerns over mistakes and doubts about actions. These three measures of maladaptive perfectionism were positively correlated with the maladaptive emotion regulation strategies (suppression and brooding) and the distress outcomes (anxiety, stress, depression, and negative affect over the past week). These correlational findings provide support for Hypothesis 1A, which was that trait levels of maladaptive perfectionism would be positively related to one's recent distress. Discrepancy and doubts about actions, but not concerns over mistakes, were negatively associated with positive affect over the past week. The three measures of maladaptive perfectionism were not associated with one's typical use of reappraisal.

The box with the large, thick dashed lines shows the correlations between the typical measures of maladaptive emotion regulation and distress. The maladaptive emotion regulation strategies (suppression and brooding) were positively associated with some of the distress outcomes (anxiety, depression, and negative affect). Brooding was associated with stress on the DASS, but suppression was not. Reappraisal was not associated with any of the distress measures and was only associated with positive affect. 
Table 3

Correlations among Scores on Perfectionism, Emotion Regulation, and Distress Measures

\begin{tabular}{|c|c|c|c|c|c|c|}
\hline & 1 & 2 & 3 & 4 & 5 & 6 \\
\hline 1. APS-R-Discrepancy & - & & & & & \\
\hline 2. FMPS-Concerns & $.59 * * *$ & - & & & & \\
\hline 3. FMPS-Doubts & $.62 * * *$ & $.50 * * *$ & - & & & \\
\hline 4. ERQ-Reappraisal & -.13 & -.02 & -.00 & - & & \\
\hline 5. ERQ-Suppression & $.41 * * *$ & $.38 * * *$ & $.35 * * *$ & .09 & - & \\
\hline 6. RRS-Brooding & $.39 * * *$ & $.34 * * *$ & $.50 * * *$ & -.03 & .14 & - \\
\hline 7. DASS-Anxiety & $.42 * * *$ & $.30 * * *$ & $.43 * * *$ & | -.02 & $.22 *$ & $.51 * * *$ \\
\hline 8. DASS-Stress & $.41 * * *$ & $.32 * * *$ & $.43 * * *$ & .05 & .17 & $.61 * * *$ \\
\hline 9. DASS-Depression & $.52 * * *$ & $.37 * * *$ & $.47 * * *$ & .02 & $.25^{* *}$ & $.58 * * *$ \\
\hline 10. PANAS-W-PA & $-.31 * * *$ & -.09 & $-.21 *$ & $.34 * * *$ & -.11 & $-.18^{*}$ \\
\hline 11. PANAS-W-NA & $.44 * * *$ & $.35 * * *$ & $.49 * * *$ & -.04 & $.18^{*}$ & $.56 * * *$ \\
\hline 12. Momentary Suppression & .03 & .08 & .01 & .06 & .07 & -.02 \\
\hline 13. Momentary Reappraisal & -.16 & -.12 & -.06 & .13 & $-.25^{*}$ & -.08 \\
\hline 14. Momentary Rumination & $.21 *$ & $.32 * * *$ & $.27 * *$ & .09 & .12 & .17 \\
\hline 15. State APS-R-Discrepancy & .06 & $.25 * *$ & .10 & .00 & .10 & .12 \\
\hline 16. State FMPS-Concerns & $.21 *$ & $.38 * * *$ & $.27 * *$ & .06 & .15 & $.24 * *$ \\
\hline 17. State FMPS-Doubts & 15 & $.30 * * *$ & $.24 * *$ & -.01 & $.17 *$ & $.20 *$ \\
\hline 18. PANAS-M-PA & -.16 & $-.23 * *$ & -.15 & .15 & -.12 & -.16 \\
\hline 19. PANAS-M-NA & $.32 * * *$ & $.36 * * *$ & $.25 * *$ & .04 & $.18 *$ & $.28 * *$ \\
\hline
\end{tabular}

(Table Continues) 
Table 3, Continued

Correlations among Scores on Perfectionism, Emotion Regulation, and Distress Measures

\begin{tabular}{|c|c|c|c|c|c|c|}
\hline & 7 & 8 & 9 & 10 & 11 & 12 \\
\hline 7. DASS-Anxiety & - & & & & & \\
\hline 8. DASS-Stress & $.75 * * *$ & - & & & & \\
\hline 9. DASS-Depression & $.62 * * *$ & $.67 * * *$ & - & & & \\
\hline 10. PANAS-W-PA & -.16 & $-.22 *$ & $-.29 * *$ & - & & \\
\hline 11. PANAS-W-NA & $.72 * * *$ & $.68 * * *$ & $.59 * * *$ & -.14 & - & \\
\hline 12. Momentary Suppression & -.01 & .09 & -.03 & .18 & .02 & - \\
\hline 13. Momentary Reappraisal & -.07 & -.07 & -.10 & $.18^{*}$ & -.08 & $.49 * * *$ \\
\hline 14. Momentary Rumination & $.19 *$ & $.21 *$ & .14 & .04 & $.25 * *$ & $.30 * * *$ \\
\hline 15. State APS-R-Discrepancy & .07 & .14 & .06 & .02 & $.18^{*}$ & $.34 * * *$ \\
\hline 16. State FMPS-Concerns & $.18^{*}$ & .16 & .15 & .05 & $.32 * * *$ & $.22 *$ \\
\hline 17. State FMPS-Doubts & .11 & $.18^{*}$ & $.18^{*}$ & .04 & $.19^{*}$ & $.35 * * *$ \\
\hline 18. PANAS-M-PA & -.16 & -.15 & -.16 & $.43 * * *$ & -.10 & .03 \\
\hline 19. PANAS-M-NA & $.31 * * *$ & $.34 * * *$ & $.31 * * *$ & .06 & $.56 * * *$ & .12 \\
\hline
\end{tabular}

(Table Continues) 
Table 3, Continued

Correlations among Scores on Perfectionism, Emotion Regulation, and Distress Measures

\begin{tabular}{|c|c|c|c|c|c|c|c|}
\hline & 13 & 14 & 15 & 16 & 17 & 18 & 19 \\
\hline 13. Momentary Reappraisal & - & & & & & & \\
\hline 14. Momentary Rumination & -.01 & - & & & & & \\
\hline 15. State APS-R-Discrepancy & .02 & $.70 * * *$ & - & & & & \\
\hline 16. State FMPS-Concerns & -.01 & $.62 * * *$ & $.68 * * *$ & - & & & \\
\hline 17. State FMPS-Doubts & -.03 & $.71 * * *$ & $.65 * * *$ & $.59 * * *$ & - & & \\
\hline 18. PANAS-M-PA & $.26 * *$ & $-.27 * *$ & $-.19 *$ & $-.17 *$ & $-.25 * *$ & - & \\
\hline 19. PANAS-M-NA & $-.18 *$ & $.43 * * *$ & $.38 * * *$ & $.51 * * *$ & $.39 * * *$ & -.05 & - \\
\hline
\end{tabular}

Note. APS-R = Almost Perfect Scale-Revised; FMPS = Frost Multidimensional Perfectionism Scale ERQ = Emotion Regulation Questionnaire; RRS = Ruminative Responses Scale; DASS = Depression Anxiety Stress Scale; PANAS-W = Positive and Negative Affect Scale-Week; PANAS-M = Positive and Negative Affect Scale-Moment; PA = Positive Affect; NA = Negative Affect, $N=136$.

$* p<.05 . * * p<.01 . * * * p<.001$

There were also positive correlations among the state measures of maladaptive perfectionism, momentary maladaptive emotion regulation, and momentary distress (see boxes with dotted lines). Measures of momentary use of suppression were positively correlated with momentary reappraisal and momentary rumination. Momentary use of reappraisal and momentary rumination were not associated. The two momentary measures of maladaptive emotion regulation strategies, suppression and rumination, were positively associated with the 
state measures of maladaptive perfectionism (discrepancy, concerns over mistakes, and doubts about actions). Momentary rumination was positively associated with negative affect and negatively associated with positive affect, whereas the inverse was true for reappraisal. Momentary suppression was not associated with either measure of affect. Reappraisal was not associated with state levels of perfectionism.

Contrary to expectations, only some of the measures of typical maladaptive perfectionism were positively correlated with their related state measures of maladaptive perfectionism (see box with thin, small dashed lines). Typical discrepancy was only positively associated with the state measure of concerns over mistakes, but it was not associated with the state measures of discrepancy or doubts about actions. Concerns over mistakes (trait) was associated with all three state measures of maladaptive perfectionism. Doubts about actions (trait) was associated with the state measures of concerns over mistakes and doubts about actions but not discrepancy. Due to the lack of expected correlations between the validated measures of trait perfectionism and the measures of state perfectionism that were constructed for this study, the measures of state perfectionism were not used in the analyses of the momentary distress model.

Similarly, most of the measures of typical emotion regulation were not correlated with the related measures of momentary emotion regulation constructed for this study (see box with thick black lines). Typical use of suppression was negatively associated with momentary use of reappraisal. However, typical suppression was not associated with momentary suppression or momentary rumination. Further, typical reappraisal and typical brooding were not associated with any of the momentary measures of emotion regulation. 


\section{Structural Equation Model of Trait Measures}

Figure 2 shows the general pathway model between maladaptive perfectionism, emotion regulation, and distress. In the structural equation model, there were two latent variables:

maladaptive perfectionism and distress. The arrows pointing from maladaptive perfectionism to the three variables below it indicate that maladaptive perfectionism is a latent variable constructed by these three observed variables. Thus, maladaptive perfectionism was measured by the Discrepancy subscale of the APS-R, the Doubts about Actions subscale of the F-MPS, and the Concerns over Mistakes subscale of the F-MPS. Similarly, the three arrows pointing from distress towards the three variables labeled anxiety, depression, and stress indicate that distress is a latent variable constructed by these three observed variables. Distress was measured by the Depression, Anxiety, and Stress subscales of the DASS-21.

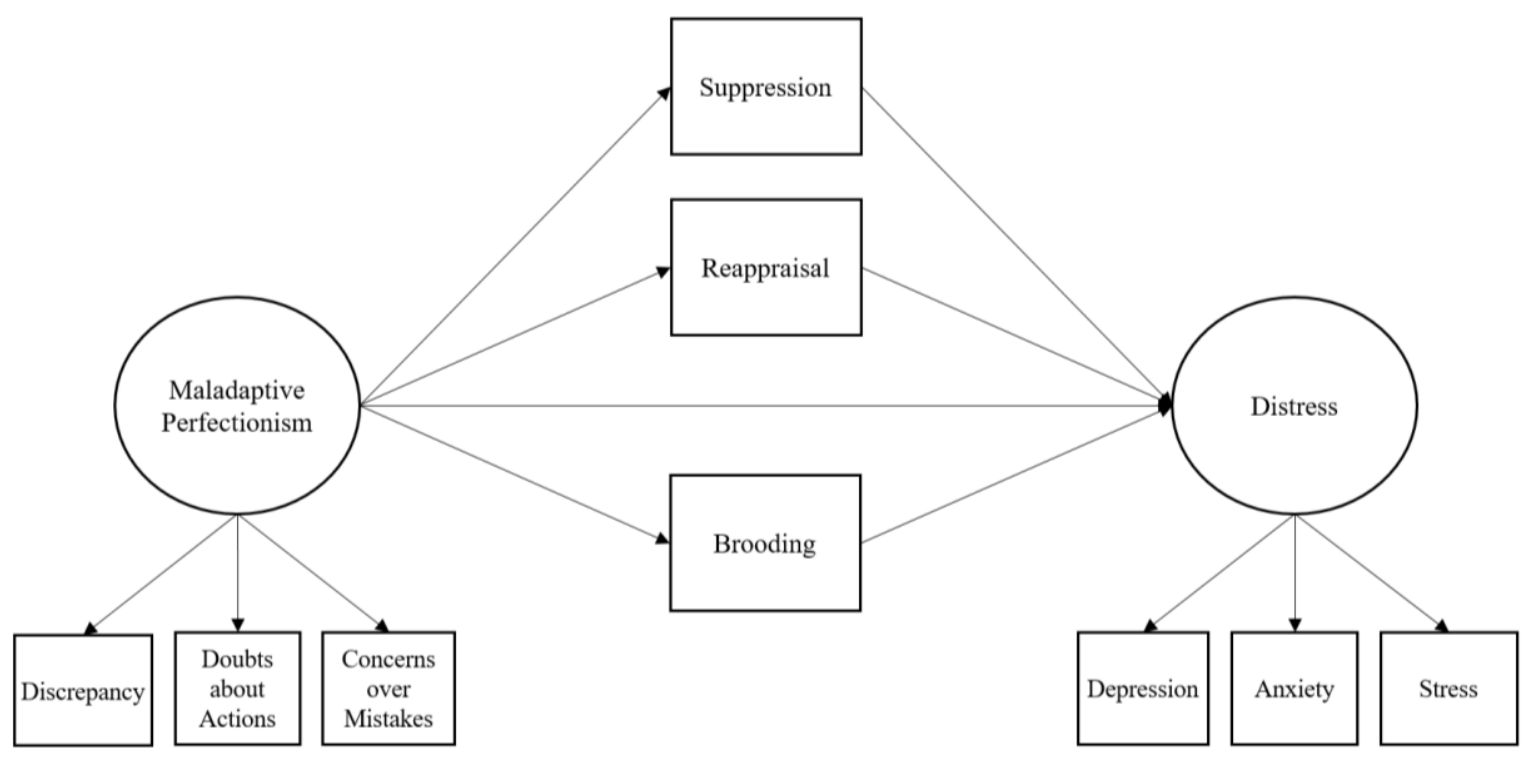

Figure 2. The conceptual model of the mediation analysis of typical maladaptive perfectionism, emotion regulation, and distress. 
Three measured variables were examined as mediators of the relation between maladaptive perfectionism and distress. These three measured variables were the Suppression subscale of the ERQ, the Reappraisal subscale of the ERQ, and the Brooding subscale of the RRS. In the conceptual model in Figure 2, the arrows point from maladaptive perfectionism to the measure of emotion regulation to distress. This demonstrates that distress was regressed upon maladaptive perfectionism and the emotion regulation strategies were used as mediators to explain this relationship.

For the structural equation model of the trait measures of maladaptive perfectionism, emotion regulation, and distress, I used four fit indices: the chi-square $\left(\chi^{2}\right)$ statistic, the Comparative Fit Index (CFI), the root mean square error of approximation (RMSEA), and the standardized root mean square residual (SRMR; Weston \& Gore, 2006). These four indices evaluate how well the estimated model fits the data. The CFI examines how the model improved over the null model in which none of the variables are related. The CFI provides a value between 0 and 1 with values greater than .95 reflecting adequate fit. The RMSEA focuses on estimated population fit. Values of .06 or less reflect adequate fit between the data and the model-implied correlations. Finally, the SRMR examines the average distance between the actual correlations in the data and the model implied correlations. If the average distance is .08 or less, the model is considered to have acceptable fit.

I used the lavaan package in $\mathrm{R}$ to examine the fit of my model using these four fit indices. The chi-square statistic indicated that the data were not significantly different from the model, $\chi^{2}(23, N=136)=34.04, p=.07$. The three other fit indices indicate a good fit between the observed data and the model $(\mathrm{CFI}=.98, \mathrm{RMSEA}=.059, \mathrm{SRMR}=.05)$. Figure 3 shows the factor loadings for the two latent variables in the model. The factor loadings of the three 
subscales on the latent variable of maladaptive perfectionism were $.83, .68$, and .76 , respectively. All three of these factor loadings were strong and significant. The factor loadings of the three subscales on the latent variable of distress were $.78, .82$, and .89 , respectively. All three of these factor loadings were also strong and significant.

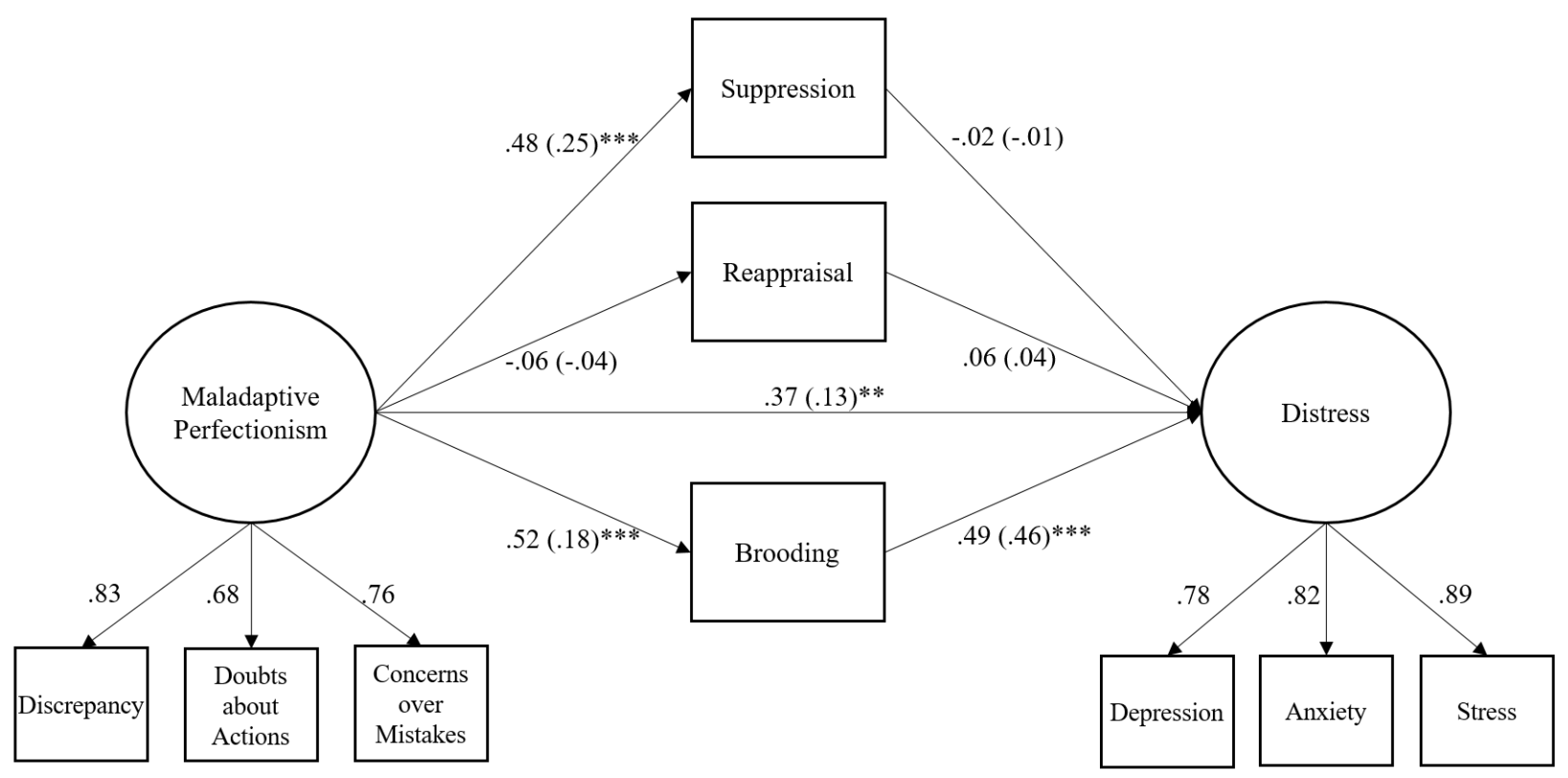

Figure 3. Factor loadings and standardized regression coefficients in the model of the mediation analysis of typical maladaptive perfectionism, emotion regulation, and distress. Unstandardized regression coefficients are shown in parentheses.

$* * p<.01 . * * * p<.001$.

I first examined the pathways between maladaptive perfectionism and each emotion regulation strategy. The pathway between maladaptive perfectionism and brooding was significant, $b=.18, t(134)=4.75, p<.001$. Maladaptive perfectionism also predicted one's typical use of suppression, $b=.25, t(134)=5.27, p<.001$. Maladaptive perfectionism did not predict reappraisal, $b=-.04, t(134)=-.59, p=.56$. 
Next, I examined the pathways between the three emotion regulation strategies and distress, as well as the pathway between maladaptive perfectionism and distress. Brooding significantly predicted distress when controlling for maladaptive perfectionism, suppression, and reappraisal, $b=.46, t(131)=5.09, p<.001$. However, suppression did not predict distress when controlling for maladaptive perfectionism, brooding, and reappraisal, $b=-0.01, t(131)=-.24, p=$ .81. The pathway between reappraisal and distress, controlling for maladaptive perfectionism, suppression, and brooding, was also not statistically significant, $b=.04, t(131)=.82, p=.41$. Finally, maladaptive perfectionism predicted distress while controlling for reappraisal, suppression, and brooding, $b=.13, t(131)=2.87, p=.004$.

Next, I tested Hypothesis 1B, which is that one's typical use of maladaptive emotion regulation strategies (that is, higher suppression, higher rumination, and lower reappraisal) would mediate the relation between maladaptive perfectionism and distress. For each of the three indirect effects, I used bootstrapping to determine the significance of the indirect effects. The process of bootstrapping involves drawing, with replacement, 10,000 samples of $N=136$ from the sample of 136 participants, which is used as a population reservoir. The lavaan package computed the unstandardized indirect effects of each bootstrapped sample. These 10,000 samples were used to determine a $95 \%$ confidence interval. This process was done for each of the three mediators: rumination (brooding), suppression, and reappraisal.

The first mediator I evaluated was brooding (see Figure 3). The indirect effect through brooding is the product of the regression coefficient for the path between maladaptive perfectionism and brooding and the regression coefficient for the path between brooding and distress. The bootstrapped, unstandardized indirect effect of maladaptive perfectionism on 
distress through brooding was .08 . The bootstrapped 95\% confidence interval ranged from .05 to .15. Therefore, this indirect effect was statistically significant.

The second mediator I evaluated was suppression. The bootstrapped, unstandardized indirect effect of maladaptive perfectionism on distress through suppression was -.003. This indirect effect was not statistically significant with the $95 \%$ confidence interval ranging from .03 to .02 . Finally, I evaluated reappraisal as a mediator. The bootstrapped, unstandardized indirect effect of maladaptive perfectionism on distress through reappraisal was -.001. This was not statistically significant with the bootstrapped $95 \%$ confidence interval ranging from -.02 to .003. In summary, Hypothesis 1B was partially supported as brooding mediated the relation between maladaptive emotion regulation and distress, but suppression and reappraisal did not.

To determine if these indirect effects were significantly different from one another, I set up three contrasts using the lavaan package in $\mathrm{R}$. The first contrast compared the indirect effect through brooding with the indirect effect through suppression. The difference between these two indirect effects was .09 with the $95 \%$ confidence interval ranging from .05 to .15 , indicating that these two indirect effects were significantly different. The second contrast examined whether the indirect effect through suppression was different from the indirect effect through reappraisal. These two indirect effects were not significantly different. The contrast was -.002 with the $95 \%$ confidence interval ranging from -.03 to .02. Finally, the third contrast was between indirect effects through reappraisal and brooding. The contrast was estimated at -.09 with the $95 \%$ confidence interval ranging from -.15 to -.05. This indicates that these two indirect effects were significantly different from one another. Thus, the indirect effect through brooding was significantly stronger than the indirect effects through suppression and reappraisal. 


\section{Moderated-Mediation of Momentary Distress}

In the momentary distress model, I intended to use a state measure of perfectionism to assess how one's perfectionistic traits manifested during the Thorndike Intelligence Measure. However, the analysis of correlations (see Table 3) demonstrated that the state measures of perfectionism were not strongly associated with the validated trait measures of perfectionism (the APS-R and the F-MPS). The lack of consistent correlations implies that the measure of state perfectionism did not accurately measure the construct that I hoped to measure, which was a manifestation of one's perfectionistic traits during the completion of the task. Due to this, I decided to remove the state measures of perfectionism from my data analysis, replacing them with the trait measures, which are validated measures of the constructs I intended to study.

Further, the measure of one's momentary use of emotion regulation strategies, the Strategies Questionnaire, was also created for this study. This questionnaire was not strongly correlated with the validated measures of typical emotion regulation (the ERQ and the RRS). However, because of the importance of assessing in-the-moment emotion regulation, the Strategies Questionnaire was still used as the measure of momentary emotion regulation in the analyses below. This was the only measure of momentary emotion regulation that the participants completed to report on their emotional response to the anagram task, which meant that although it was not correlated with the validated emotion regulation measures, it was the only option that I could use for the mediation analysis.

To conduct my analyses for Hypothesis $2 \mathrm{~A}$ and $2 \mathrm{~B}$, I created three models of moderated mediation for each of the three mediators I examined: rumination, suppression, and reappraisal. I created three separate models, as opposed to using structural equation modeling with three mediators and three moderators, to reduce the complexity of the analyses. Figure 4 shows the 
general pattern of each of these mediation models. In each of the three models, maladaptive perfectionism was defined as the average of the participant's standardized scores across the three trait measures of maladaptive perfectionism. Thus, it was not treated as a latent variable. Further, the momentary use of each of the three emotion regulation strategies was examined as a mediator between maladaptive perfectionism and distress. The arrow pointing from maladaptive perfectionism towards the emotion regulation strategy and the arrow pointing from the emotion regulation strategy to in-the-moment negative affect indicate the mediation. The arrow pointing from the failure condition towards the path between maladaptive perfectionism and the emotion regulation strategy represents that the failure condition was examined as a moderator of this pathway. The moderator was a dichotomous variable with the low-failure condition being coded as 0 and the high-failure condition being coded as 1. Finally, the arrow pointing from negative affect over the past week towards negative affect in the moment shows that negative affect over the past week was used as a control variable for in-the-moment negative affect.

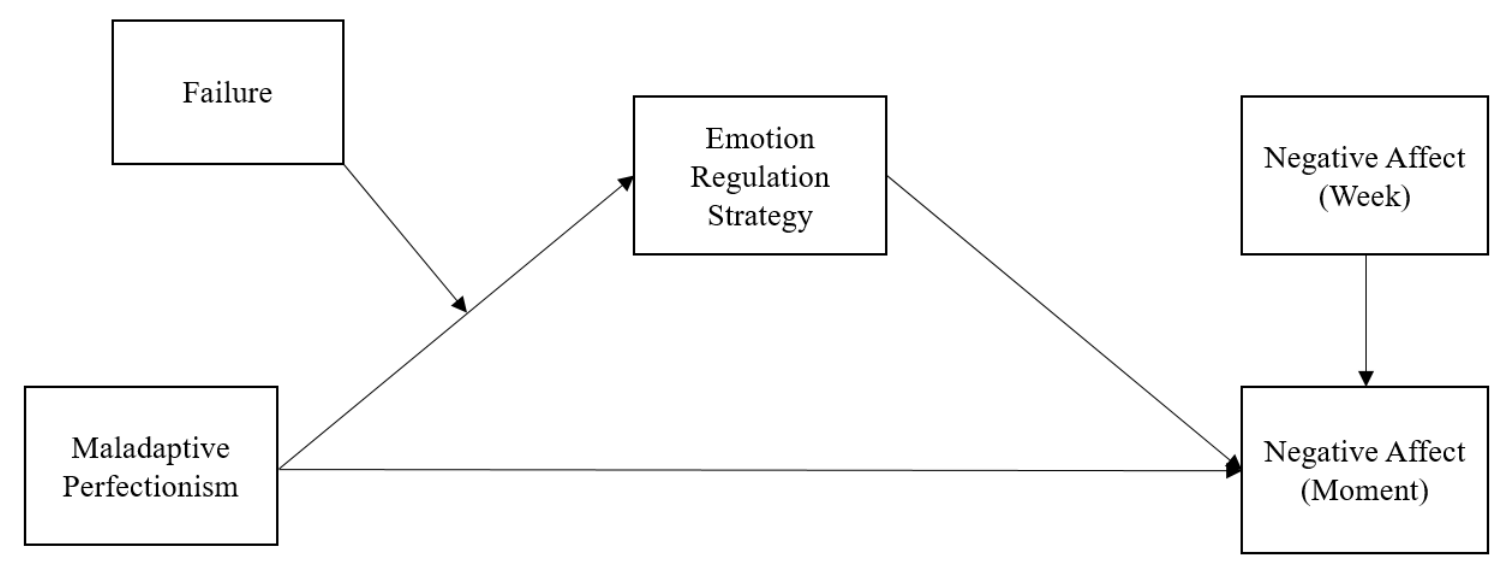

Figure 4. Conceptual diagram of the momentary distress model of moderated mediation. Three emotion regulation strategies (rumination, suppression, reappraisal) were used as mediators in three separate models. 
First, I examined the moderated mediation model for rumination, which is displayed in Figure 5. The failure condition moderated the pathway between maladaptive perfectionism and rumination, $b=-2.18, t(132)=-2.20, p=.03$. The simple slope between maladaptive perfectionism and momentary rumination was significant for the low-failure condition, $b=2.71$, $t(132)=4.13, p<.001$. The simple slope between maladaptive perfectionism and momentary rumination for the high-failure condition was not significantly different from zero, $b=.53, t(132)$ $=.90, p=.37$. Momentary rumination significantly predicted negative affect when controlling for maladaptive perfectionism and negative affect over the past week, $b=.41, t(132)=3.69, p<$ .001 .

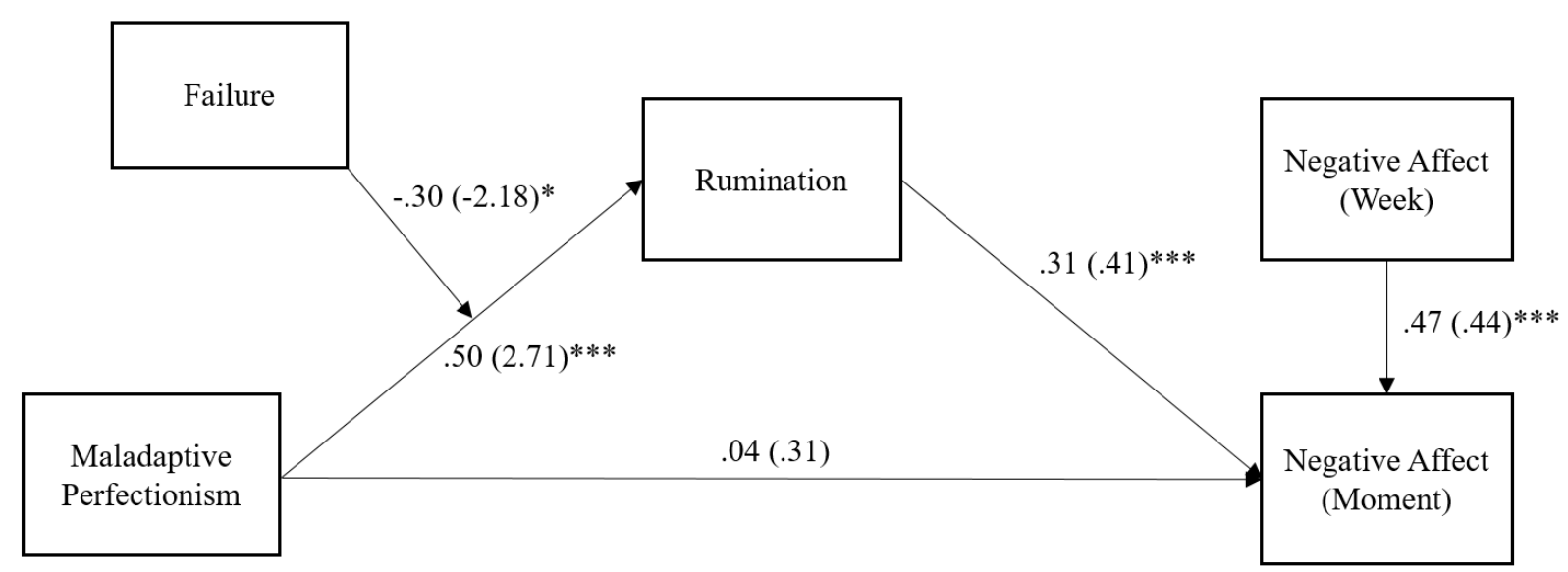

Figure 5. Standardized regression coefficients for the moderated mediation model for rumination are shown. Unstandardized regression coefficients are in parentheses. $* p<.05 . * * * p<.001$

For the indirect effect through rumination, I computed the product of the regression coefficient for the path between maladaptive perfectionism and rumination and the regression 
coefficient for the path between rumination and distress for both conditions. As before, I used bootstrapping to test the indirect effects. The indirect effect of maladaptive perfectionism on distress through rumination for the low-failure condition was 1.11 , which was statistically significant with the $95 \%$ bootstrapped confidence interval ranging from .49 to 2.03 . Therefore, this indirect effect was statistically significant. For the high-failure condition, the bootstrapped, unstandardized indirect effect was .22 . The $95 \%$ bootstrapped confidence interval ranged from .35 to 1.07 , which indicates that the indirect effect was not significant for the high failure group. The index of moderated mediation shows whether or not moderated mediation is present by quantifying the weight of the moderator (failure condition) on the indirect effect of maladaptive perfectionism on distress through rumination. The index of moderated mediation for this model was -.90 , which was statistically significant as evidenced by the confidence interval ranging from -1.92 to -.18. Although moderation was present, Hypothesis $2 \mathrm{~A}$ and $2 \mathrm{~B}$ were not supported for rumination because the indirect effect was significant for the low-failure condition rather than the high-failure condition. Hence, the moderation affected the indirect effect in the opposite direction from what I expected. The chi-square statistic indicated that the data were not significantly different from the model, $\chi^{2}(3, N=136)=5.48, p=.14$. Further, the SRMR (.03) and the CFI (.97) demonstrated adequate fit. However, the RMSEA of .08 did not indicate adequate fit.

Figure 6 shows the moderated mediation model for suppression, which is the model I examined next. Maladaptive perfectionism did not predict momentary suppression in the lowfailure condition, $b=.71, t(132)=1.11, p=.27$. Maladaptive perfectionism also did not predict momentary suppression in the high-failure condition, $b=-.31, t(132)=-.55, p=.58$. Momentary suppression did not significantly predict negative affect when controlling for maladaptive 
perfectionism and negative affect over the past week, $b=.17, t(132)=1.52, p=.13$. Further, the experience of failure did not moderate the pathway between maladaptive perfectionism and suppression, $b=-1.02, t(132)=-.96, p=.34$.

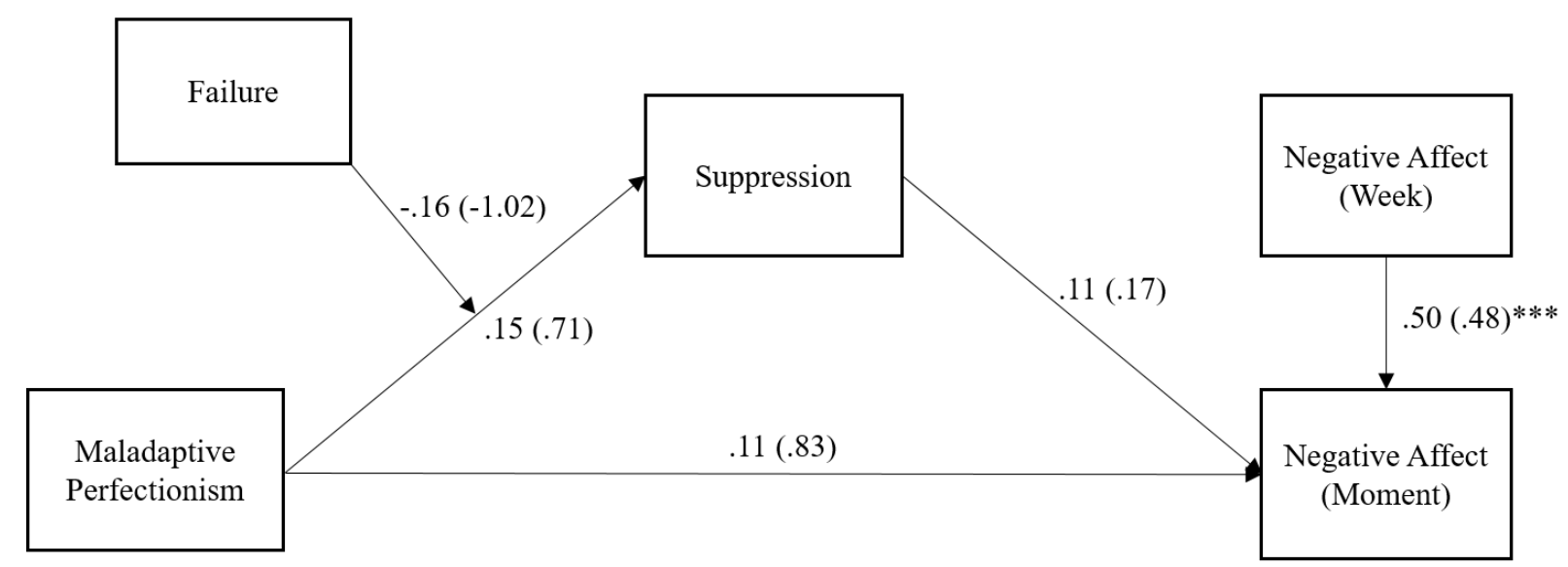

Figure 6. Standardized regression coefficients for the moderated mediation model for suppression are shown. Unstandardized regression coefficients are in parentheses. $* * * p<.001$.

The bootstrapped, unstandardized indirect effect of maladaptive perfectionism on distress through suppression for the low-failure condition was .12. This was not statistically significant as evidenced by the confidence interval ranging from -.08 to .66 . The bootstrapped, unstandardized indirect effect of maladaptive perfectionism on distress through suppression for the high-failure condition was -.05. This also was not statistically significant as evidenced by the confidence interval ranging from -.49 to .16 .

The index of moderated mediation was -.17, which was not statistically significant with the confidence interval ranging from -.91 to .11. Hence, Hypothesis $2 \mathrm{~A}$ and $2 \mathrm{~B}$ were not supported for suppression. The chi-square statistic indicated that the data was not significantly different from the model, $\chi^{2}(3, N=136)=7.60, p=.06$. The SRMR of .04 demonstrated 
adequate fit. However, the two other fit indices indicated that there was not adequate fit between the observed data and the model, $\mathrm{CFI}=.92, \mathrm{RMSEA}=.11$.

Finally, I examined the moderated mediation model for reappraisal, which can be seen in Figure 7. The pathway between maladaptive perfectionism and reappraisal was not statistically significant for the low-failure condition, $b=.27, t(132)=.48, p=.64$. However, the pathway between maladaptive perfectionism and reappraisal was statistically significant for the highfailure condition, $b=-1.16, t(132)=-2.30, p=.02$. The pathway between reappraisal and negative affect, controlling for maladaptive perfectionism and negative affect over the past week, also was not significant, $b=-.22, t(132)=-1.67, p=.10$. The experience of failure did not moderate the pathway between maladaptive perfectionism and reappraisal, $b=-1.43, t(132)=-$ $1.61, p=.11$.

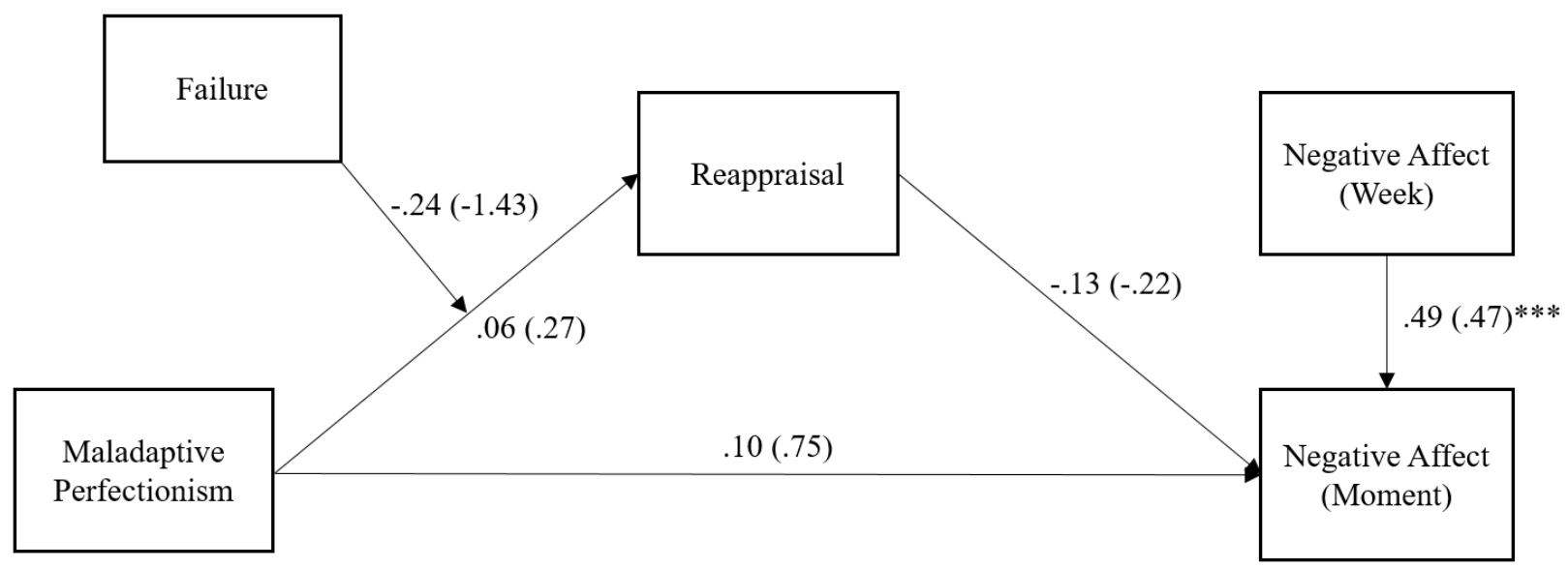

Figure 7. Standardized regression coefficients for the moderated mediation model for reappraisal are shown. Unstandardized regression coefficients are in parentheses.

$* * * p<.001$.

The bootstrapped, unstandardized indirect effect of maladaptive perfectionism on distress through suppression was -.06 for the low-failure condition with the $95 \%$ confidence interval 
ranging from -.53 to .22 . Therefore, this indirect effect was not statistically significant. The bootstrapped, unstandardized indirect effect of maladaptive perfectionism on distress through suppression was .26 for the high-failure condition with the $95 \%$ confidence interval ranging from -.01 to .85 , which also indicates that the indirect effect was not statistically significant.

The index of moderated mediation was .32 with the confidence interval ranging from -.02 to 1.10 . Therefore, the index of moderated mediation was not statistically significant. Hence, Hypothesis $2 \mathrm{~A}$ and $2 \mathrm{~B}$ were not supported for reappraisal. The chi-square statistic indicated that the data were significantly different from the model, $\chi^{2}(3, N=136)=9.23, p=.03$. The SRMR demonstrated adequate fit, SRMR $=.04$. However, the two other fit indices indicated that there was not adequate fit between the observed data and the model, $\mathrm{CFI}=.90, \mathrm{RMSEA}=.12$. 


\section{CHAPTER V: DISCUSSION}

Research has demonstrated that maladaptive emotion regulation mediates the relation between maladaptive perfectionism and distress (Castro et al., 2017; Macedo et al., 2017; O’Connor et al., 2007). This mediation model has not been examined in a laboratory setting when individuals face an experience of failure. I conducted an experimental study in which participants experienced two levels of failure in the form of an intelligence test. Through this study, I confirmed the findings from previous research that, in the general model, rumination mediated the relation between maladaptive perfectionism and distress. Suppression and reappraisal did not mediate this relation. I found that in the momentary distress model, rumination mediated the relation between maladaptive perfectionism and distress (i.e., negative affect) in the low-failure condition but not in the high-failure condition. I found that moderated mediation was not present for suppression or reappraisal.

\section{General Model}

Hypothesis 1 was that one's typical use of maladaptive emotion regulation strategies (higher suppression, higher rumination, and lower reappraisal) would mediate the positive relation between trait levels of maladaptive perfectionism and one's recent (i.e., past week) level of distress. Multiple studies have shown that the brooding component of rumination mediated the relation between maladaptive perfectionism and distress-related outcomes such as depression, anxiety, and somatic symptoms (Di Schiena et al., 2012; Macedo et al., 2017; O'Connor et al., 2007; Senra et al., 2017). The present study confirmed this model. Hence, rumination helps us understand why perfectionism is associated with negative outcomes such as depression, anxiety, and stress. More specifically, the brooding component of rumination plays an important role in these outcomes. Brooding has to do with reflecting on one's negative emotions in a repetitive, 
anxious manner (Treynor et al., 2003). Individuals with high levels of maladaptive perfectionism seem to experience more negative outcomes because they turn their focus inward with selfcriticism and self-blame, which are features of brooding.

Suppression and reappraisal did not mediate the relation between maladaptive perfectionism and distress in the general model. There are two important points to be made when examining these findings. First, these analyses were conducted as a multiple mediation model, meaning that when each of the mediators was analyzed, the other mediators were held constant. This means that suppression and reappraisal did not explain the relation between maladaptive perfectionism and distress above and beyond the explanatory power of rumination. In other words, the way that an individual with maladaptive perfectionism turns their focus to their negative emotional response by dwelling on their disappointment contributes more to their distress than their acts of expressive suppression or their lack of reappraisal. Second, the pathway between maladaptive perfectionism and distress was still statistically significant after taking into account the effects of the three mediators. This demonstrates that maladaptive perfectionism may be related to distress through some other explanatory factor beyond the three emotion regulation strategies considered in these analyses.

As mentioned above, suppression did not mediate the relation between maladaptive perfectionism and distress in the general model. Maladaptive perfectionism predicted suppression, which mirrors previous research findings (Bergman et al., 2007; Richardson et al., 2014). However, in the present study, suppression did not predict the negative outcomes of depression, anxiety, and stress while controlling for maladaptive perfectionism, rumination, and reappraisal. This finding contradicts previous research that showed that suppression was positively related to depressive symptoms, decreased self-esteem, and increased negative 
emotion (Gross \& John, 2003). However, the present finding is likely due to the fact that in this analysis, each of the mediators acted as control variables for one another. This finding demonstrates that suppression has less of a role in explaining the negative outcomes of maladaptive perfectionism when compared to other emotion regulation strategies.

Reappraisal also did not mediate the relation between maladaptive perfectionism and distress in the general model. Maladaptive perfectionism did not predict the use of reappraisal, and reappraisal did not predict distress. Reappraisal, an adaptive emotion regulation strategy, has to do with the process of using cognitive change to alter one's perception of the situation causing emotional distress to decrease negative emotions (Gross, 1998). There is limited research on the relation between reappraisal and maladaptive perfectionism, but one study found that reappraisal mediated the relation between concerns over mistakes and negative affect (Castro et al., 2017).

One explanation for the lack of mediation through reappraisal is that perhaps the high use of rumination among individuals high in maladaptive perfectionism interferes with their ability to effectively use reappraisal. It is possible that even when these individuals try to change their perspective on the situation using reappraisal, their constant ruminative tendencies prevent these attempts from making lasting cognitive change. Malivoire and colleagues (2019) explained that maladaptive perfectionists may struggle to change their rigid thinking styles, which supports this idea. Future research could confirm this idea by examining a serial mediation model. For example, one could examine if perfectionism predicts increased rumination, which then predicts reduced reappraisal, which in turn predicts increased distress.

Another possible explanation for why one's use of reappraisal did not explain the relation between maladaptive perfectionism and distress in this study is that rumination has a stronger explanatory role. However, it is still unlikely that mediation through reappraisal would occur 
because the zero-order correlations between trait levels of maladaptive perfectionism and momentary reappraisal were not statistically significant (see Table 3).

\section{Momentary Distress Model}

There has been limited research on how this mediation model arises in the moment when an individual faces a distressing situation. Van der Kaap-Deeder and colleagues (2016) found that a week after an experience of failure, individuals with maladaptive perfectionism used maladaptive emotion regulation strategies, such as rumination, when reflecting on the experience of failure. Another study found that maladaptive perfectionists experienced distress after one experience of failure and felt increased distress after multiple experiences of failure (Stoeber, Schneider, Hussain, \& Matthews, 2014). Hypothesis $2 \mathrm{~A}$ was that, in a situation that induces an extreme feeling of failure, momentary use of maladaptive emotion regulation strategies (i.e., higher suppression, higher rumination, lower reappraisal) would mediate the relation between maladaptive perfectionism and momentary distress with typical distress being controlled.

The present study revealed that, in the momentary distress model, rumination was the only emotion regulation strategy that mediated the relation between maladaptive perfectionism and distress, with mediation being present in the low-failure condition but not the high-failure condition. This finding partially supports my hypothesis that the general mediation model seen in the literature would be present in the moment when an individual with high levels of maladaptive perfectionism faces failure. However, the mediation appeared in the low-failure condition rather than the high-failure condition.

Rumination is the act of focusing attention and reflecting on one's negative mood in an anxious or gloomy manner (Treynor et al., 2003). The use of rumination is associated with negative outcomes such as depressive episodes and anxiety (Nolen-Hoeksema, 2000). It appears 
that this particular emotion regulation strategy has an important role in how individuals with maladaptive perfectionism respond to experiences of failure. Namely, they mull over their mistakes and frustration about their performance.

This pattern may have to do with the measures of maladaptive perfectionism used in this study. High scores on the Discrepancy subscale of the APS-R represent dissatisfaction in one's performance in relation to one's high standards (Flett et al., 2016). High scores on the Doubts about Actions and Concerns over Mistakes subscales of the F-MPS are associated with selfcritical aspects of depression (Frost \& Marten, 1990). It is understandable that participants with high scores on these scales associated with self-critical evaluation would reflect inwardly in a ruminative, anxious manner when they perceived falling short of standards. The act of ruminating on this perceived failure would be a plausible result of these self-evaluative perfectionistic traits, which would understandably lead individuals with these traits to experience increased distress.

Hypothesis $2 \mathrm{~B}$ was that the indirect effect of maladaptive perfectionism on distress through maladaptive emotion regulation would be greater in the high-failure condition than the low-failure condition. I found that moderated mediation was present in the momentary distress model with rumination as the mediator. However, the indirect effect of maladaptive perfectionism on distress through rumination was greater in the low-failure condition than in the high-failure condition, which was contrary to my hypothesis. Further, the indirect effect was only significant in the low-failure condition and not the high-failure condition.

These results demonstrate that maladaptive perfectionism is particularly important at relatively low or moderate levels of distress (i.e., low failure), rather than in situations that evoke extreme distress (i.e., high failure). It appears that when individuals with maladaptive 
perfectionism feel like they have fallen short of standards that are somewhat attainable or within their reach, they respond in a way that leads them to have negative affect and feel distressed. This supports other research that shows that individuals with maladaptive perfectionism used rumination and felt distressed following experiences of presumably moderate, rather than extreme, failure (Besser et al., 2004; Van der Kaap-Deeder et al., 2016).

However, when the standard feels excessively out of reach and one's performance falls extremely short of this standard, this mediation model was not supported. One explanation for this finding is that when facing this extreme level of failure, individuals with maladaptive perfectionism may have decided to attribute their failure to the difficulty of the task rather than their own shortcomings. This idea is supported by the research of Stoeber and Becker (2008) who found that individuals with negative perfectionism (i.e., those who attempt to avoid imperfections and its negative outcomes) attributed their failures to external factors rather than internal factors. As the failure task in the present study got more extreme, individuals with high maladaptive perfectionism may have been more likely to place the blame of their failure on the task itself to avoid harm to their self-esteem.

A second explanation for this finding is that when the standards are excessively high and the failure is extreme, individuals with maladaptive perfectionism may reduce their rumination because they emotionally "give up." Perhaps at this level of failure, these individuals detach from the set standard in an attempt to avoid the feeling of failure and falling short. This explanation may be supported by Stoeber and colleagues' (2014) finding that, for initial failures, maladaptive perfectionism predicted increased anxiety, anger, and depression, whereas repeated failures predicted increased anger. If the participants with maladaptive perfectionism in this study felt increased anger as they continued to fail at finding anagram solutions for $10 \mathrm{~min}$, they 
may have attempted to "shut down" emotionally or separate themselves mentally from the goal to avoid this negative emotion. If it is the case that participants disengaged from the goal mentally, it was not evidenced by a difference in their effort towards the task, as there were no group-level differences in effort on the Thorndike Intelligence Measure.

This explanation may be related to a study by Lo and Abbott (2013), which found that when maladaptive perfectionists were given a task to solve unsolvable anagrams, they were more persistent in their efforts when there were lower expected standards in comparison to when the expected standards were higher. The researchers suggested that the maladaptive perfectionists may have stopped giving effort towards reaching the high standard because they felt overwhelmed and were attempting to avoid feeling inferior. This finding supports the present study's explanation that in an experience of high-failure, individuals with high maladaptive perfectionism disconnected from their goal, which led to decreased rumination and distress. This explanation may have been especially true if participants did not personally identify with the goal of scoring high on an intelligence test.

On the other hand, it is possible that rather than giving up, individuals high in maladaptive perfectionism decided to reevaluate their personal standards while completing the anagram task in the high-failure condition. In other words, these individuals may have reduced their stress by deciding that a lower standard was acceptable due to the difficulty of the task. This explanation would enhance our understanding of maladaptive perfectionism, but it also contradicts what previous research has shown about how maladaptive perfectionists reset their standards. For example, Shafran and colleagues (2002) hypothesized based on previous research that clinical perfectionism would be associated with setting higher standards for oneself after failure. In fact, Egan, Piek, Dyck, Rees, and Hagger (2013) found that some individuals with 
clinical perfectionism reset their standards to be higher following a failure experience. On the other hand, Egan, Dick, and Allen (2012) found that clinical perfectionism was not associated with how one resets his or her standards following success or failure, although they did confirm that clinical perfectionism was associated with higher standard-setting overall.

Suppression did not mediate the relation between maladaptive perfectionism and distress in the moment. Suppression is defined as one's attempt to conceal his or her emotionalexpressive behaviors (Gross, 2008). This definition implies an interpersonal component, given that suppression involves hiding one's physical expressions. In the present study, participants were not being observed by the researcher when they were completing the anagram task nor when they were completing the questionnaires. The limited interpersonal interaction may have reduced the participants' perceived need to use suppression, which therefore reduced the impact of this emotion regulation strategy on their distress. Another potential explanation for suppression's lack of influence on the model has to do with the measures of maladaptive perfectionism used in the study. The Discrepancy, Concerns over Mistakes, and Doubts about Actions subscales from the APS-R and the F-MPS are all intrapersonal measures of perfectionism (Sironic \& Reeve, 2015). Other subscales of the F-MPS and the HF-MPS are considered to assess more interpersonal aspects of perfectionism (e.g., socially prescribed perfectionism). Perhaps intrapersonal traits of perfectionism, like the ones used in this study, are more strongly associated with more cognitive emotion regulation strategies like rumination, whereas individuals high in interpersonal traits of perfectionism may use interpersonal emotion regulation strategies, like expressive suppression.

Reappraisal also did not mediate the relation between maladaptive perfectionism and distress in the moment. The reason why reappraisal did not mediate this relation may have to do 
with its placement in the modal model. Reappraisal has to do with the way that one changes their cognitions to experience less negative emotion regarding a situation (Gross, 2008). It is possible that participants did not reach this later stage in the emotion regulation process while they were still taking the test. Perhaps participants may have utilized reappraisal after time had passed and they reflected on their interpretation of the task. Another possible explanation has to do with the intensity of the emotional experience in this study. Research has shown that reappraisal is not effective or preferred when emotional intensity is high (Sheppes, Scheibe, Suri, \& Gross, 2011), which may have been the case for some participants who felt strongly affected by the experience of failure.

\section{Limitations of the Present Study}

One limitation of the present study is the measure of momentary emotion regulation that was used. The measures of momentary emotion regulation (suppression, rumination, and reappraisal) that I created for this study were not correlated with the validated measures of one's typical use of these strategies, the ERQ and the RRS. I intended to use these measures to assess how much participants ruminated, suppressed their emotions, and used reappraisal during the Thorndike Intelligence Measure. However, the lack of correlations with other measures of the same construct raises concerns about whether or not the momentary measures captured these strategies for the momentary distress model. Due to the lack of correlations, the momentary measures of emotion regulations may not have captured the entire construct of the emotion regulation strategy that it was intended to measure. Another possible explanation is that perhaps these two constructs - one's typical use of emotion regulation strategies and one's application of emotion regulation strategies towards a given task — have theoretical differences that cause them to not have stronger correlations. Perhaps the way that individuals regulate their emotions in 
response to a failure task differs from how they typically regulate their emotions in their day-today life. This lack of validity may contribute to the reason why these measures of suppression and reappraisal did not mediate the relation in the momentary distress model in the expected way. A follow-up study should be completed after addressing this problem by improving the construct validity of scores from these measures.

Similarly, future research should consider both trait perfectionism and state perfectionism when exploring general models and momentary distress models. For the present study, I intended to create a measure of state perfectionism to examine how much participants experienced feelings of discrepancy, concern over their mistakes, and doubts about their actions in the moment while completing the task. The goal of this measure was to differentiate between one's trait-levels of perfectionism and how these traits are activated when facing a task. The state perfectionism measure was created to assess the activation and application of one's perfectionistic traits in the moment. However, the constructed state-level measures of these traits were not correlated with the validated measures of these constructs, the APS-R and the F-MPS, which is a second limitation of the study. It is possible that these measures were not correlated because the subscales, with only two items each, created for the state perfectionism questionnaire did not adequately represent their respective trait-level constructs. A second explanation is that it may be difficult for participants to consciously report the manifestation of their perfectionism towards a task due to the automatic and fleeting nature of their perfectionistic thoughts. Although I was able to use the participants' trait levels of perfectionism for the momentary distress model, research would benefit from a measure of how individuals apply these traits to an experience of momentary distress, such as failure. A measure of this kind would improve our understanding of how perfectionism is experienced and applied during stress-inducing situations. Perhaps a 
measure of one's perfectionistic cognitions could be used when examining perfectionism in the laboratory. A future study should be completed using a perfectionistic cognition measure or an updated version of the state perfectionism measure with improved construct validity.

A third limitation of the present study was the difficulty of the Thorndike Intelligence Measure. It appeared that the anagram task used in the high-failure condition was excessively challenging, which contributed to the unexpected results in the moderated mediation model. This study originally intended to use failure as a moderator by having a non-failure (no distress) experience and a failure (momentary distress) experience. Due to the extreme difficulty of the measure used in the high-failure condition, the two groups became a low-failure condition (momentary distress) and a high-failure condition (extreme momentary distress). Although this is an important comparison to examine, comparing the effects of a non-failure experience with a failure experience would further contribute to the research and reveal more about these relations. A follow-up study comparing an experience of momentary distress with a group that does not experience any distress would be beneficial.

Fourth, the small sample size of this study is a limitation when doing structural equation modelling. Due to the small sample size, the $p$ values that were reported may not be trustworthy or interpretable in the structural equation model and for the chi-squared statistics in the momentary model. Future research could attempt to replicate this study with a larger sample to address this problem. A final limitation is the generalizability of these findings. First, it is possible that college students respond differently to tests of intelligence due to the increased focus on academic success in the university setting. It would be important to complete a followup study with a broader population to examine how an experience of failure affects individuals of different ages and in different settings. Further, the specific type of failure used to induce 
momentary distress may not be entirely generalizable as an experience of failure for all participants. The failure experience was designed to make participants feel as though they were falling short of a standard of intelligence. I presumed that this would feel like an internalized stressor for someone who has excessively high standards. However, the type of high standards that individuals with maladaptive perfectionism identify with may vary from person to person. Whereas some individuals may identify with high standards of intelligence, other individuals may identify with high standards of athleticism or body shape. Future research should consider what kinds of high standards participants set for themselves to ensure that the experience of failure actually causes momentary distress and induces the perception of falling short of an internalized standard.

\section{Implications}

This study has implications for future research on perfectionism. The majority of research on perfectionism focuses on trait levels of perfectionism as measured by the APS-R, F-MPS, and HF-MPS. However, there is limited research on how perfectionistic traits manifest in the moment when individuals experience a distressing situation. A state measure of perfectionism would help researchers assess how certain traits of perfectionism appear in one's moment-tomoment decision-making, behaviors, and approaches to task completion in experimental designs. To my knowledge, no previous research has attempted to assess how the existing traits of perfectionism emerge in the moment by creating state measures of these traits. Although the newly constructed measures did not demonstrate validity in the present study, this idea lays the groundwork for how future research could examine certain perfectionistic traits in a momentary distress model. 
Second, this research demonstrates the potential of using experimental designs to examine the effects of maladaptive perfectionism. Whereas the majority of research on this topic focuses on correlational designs, a few studies have found significant results when examining how perfectionism affects emotion regulation using experimental manipulation (Lo \& Abbott, 2013; Stoeber et al., 2014; Van der Kaap-Deeder et al., 2016). Further, the findings in the present study that maladaptive perfectionism was associated with momentary emotion dysregulation and distress in an experimental design demonstrate an opportunity for future research on more complex variants of these relations. For example, future research could use the moderatedmediation model to see if certain interventions affect the relation between maladaptive perfectionism and rumination such as mindfulness, social comparison, or reflection upon values. This study informs future research by demonstrating that generalized models of perfectionism can be translated to the laboratory setting.

This study also has implications for our theoretical understanding of maladaptive perfectionism, emotion regulation, and distress. First, the research on the general mediation model includes diverse definitions of all three variables in the model: maladaptive perfectionism, maladaptive emotion regulation, and distress. For instance, the examined mediators have included but are not limited to maladaptive coping mechanisms (Dunkley \& Blankenstein, 2000), rumination (O'Connor et al., 2007), catastrophizing (Macedo et al., 2017), and reappraisal (Castro et al., 2017). The present study examines these relations in a streamlined model using two measures of emotion regulation (the ERQ and the RRS) that are commonly used in research. Through this study, we learned that rumination is the most important explanatory factor in the relation between maladaptive perfectionism and distress. This informs and strengthens our understanding of how maladaptive perfectionism leads to negative outcomes. 
This research also has applied implications for the clinical treatment of individuals with perfectionism. The present study highlights the importance of rumination in the stress response of an individual with maladaptive perfectionism. Whereas rumination can hinder mental health treatment outcomes (Price \& Anderson, 2012), targeting rumination can also improve the effectiveness of certain treatments (Spinhoven et al., 2018). Given these findings, I suggest that the treatment of individuals with maladaptive perfectionism may be more effective if rumination is directly addressed.

Finally, the present study's findings suggest that individuals with maladaptive perfectionism may mentally detach from their goal when facing extreme levels of distress. Therefore, these individuals may avoid seeking mental health treatment in excessively stressful situations to avoid feeling inferior or facing failure. This problem could be addressed by following the suggestions from research on treating individuals who avoid counseling, such as by educating clients about counseling, connecting clients to community supports, and providing services in non-traditions methods such as at home or online (Vogel, Wester, \& Larson, 2007). Similarly, knowing that perfectionism is associated with self-concealment (Kawamura \& Frost, 2004), the present study suggests that individuals with maladaptive perfectionism might be even less likely to disclose extremely distressing information. If perfectionistic clients seek mental health treatment, practitioners should consider using interventions that reduce avoidance, such as the suggestions by Richardson and Rice (2015) to use mindfulness-based approaches and to encourage clients to practice sharing their daily stressors with others. 


\section{REFERENCES}

Aldea, M. A., \& Rice, K. G. (2006). The role of emotion dysregulation in perfectionism and psychological distress. Journal of Counseling Psychology, 53, 498-510.

American Psychiatric Association. (2013). Diagnostic and statistical manual of mental disorders (5th ed.). Arlington, VA: American Psychiatric Publishing.

Ashby, J. S., Rice, K. G., \& Martin, J. L. (2006). Perfectionism, shame, and depressive symptoms. Journal of Counseling \& Development, 84, 148-156.

Aspinwall, L. G., \& Richter, L. (1999). Optimism and self-mastery predict more rapid disengagement from unsolvable tasks in the presence of alternatives. Motivation and Emotion, 23, 221-245.

Berman, A. J., Nyland J. E., \& Burns, L. R. (2007). Correlations with perfectionism and the utility of a dual process model. Personality and Individual Differences, 43, 389-399.

Besser, A., Flett, G. L., Guez, J., \& Hewitt, P. L. (2008). Perfectionism, mood, and memory for positive, negative, and perfectionistic content. Individual Differences Research, 6, 211244.

Besser, A., Flett, G. L., \& Hewitt, P. L. (2004). Perfectionism, cognition, and affect in response to performance: Failure vs. success. Journal of Rational-Emotive \& Cognitive Behavior Therapy, 22, 301-328

Bieling, P. J., Israeli, A. L., \& Antony, M. M. (2004). Is perfectionism good, bad, or both? Examining models of the perfectionism construct. Personality and Individual Differences, 36, 1373-1385. 
Blatt, S. J., \& Zuroff, D. C. (2002). Perfectionism in the therapeutic process. In G. L. Flett \& P.

L. Hewitt (Eds.), Perfectionism: Theory, research, and treatment (pp. 396-406). Washington, DC: American Psychological Association.

Cabanac, M. (2002). What is emotion? Behavioural Processes, 60, 69-84.

Cackowski, J. M., \& Nasar, J. L. (2003). The restorative effects of roadside vegetation: Implications for automobile driver anger and frustration. Environment and Behavior, 35, 736-751.

Castro, J., Soares, M. J., Pereira, A. T., \& Macedo, A. (2017). Perfectionism and negative/positive affect associations: The role of cognitive emotion regulation and perceived distress/coping. Trends in Psychiatry and Psychotherapy, 39, 77-87.

Desnoyers, A., \& Arpin-Cribbie, C. (2015). Examining cognitive performance: Do perfectionism and rumination matter? Personality and Individual Differences, 76, 94-98.

Di Schiena, R., Luminet, O., Philippot, P., \& Douilliez, C. (2012). Adaptive and maladaptive perfectionism in depression: Preliminary evidence on the role of adaptive and maladaptive rumination. Personality and Individual Differences, 53, 774-778.

Dryman, M. T., \& Heimberg, R. G. (2018). Emotion regulation in social and depression: a systematic review of expressive suppression and cognitive reappraisal. Clinical Psychology Review, 65, 17-42.

Dunkley, D. M. (2018). Perfectionism and daily stress, coping, and affect. In J. Stoeber (Ed.), The Psychology of Perfectionism (pp. 222-242). New York, NY: Routledge.

Dunkley, D. M., \& Blankstein, K. R. (2000). Self-critical perfectionism, coping, hassles, and current distress: A structural equation modeling approach. Cognitive Therapy and Research, 24, 713-730. 
Dunkley, D. M., Blankstein, K. R., Halsall, J., Williams, M., \& Winkworth, G. (2000). The relation between perfectionism and distress: Hassles, coping, and perceived social support as mediators and moderators. Journal of Counseling Psychology, 47, 437-453.

Dunkley, D. M., Zuroff, D. C., \& Blankstein, K. R. (2003). Self-critical perfectionism and daily affect: Dispositional and situational influences on stress and coping. Journal of Personality and Social Psychology, 84, 234-252.

Egan, S. J., Dick, M., \& Allen, P. J. (2012). An experimental investigation of standard setting in clinical perfectionism. Behaviour Change, 29, 183-195.

Egan, S. J., Piek, J. P., Dyck, M. J., Rees, C. S., \& Hagger, M. S. (2013). A clinical investigation of motivation to change standards and cognitions about failure in perfectionism. Behavioural and Cognitive Psychotherapy, 41, 565-578.

Ehring, T., Tuschen-Caffier, B., Schnülle, J., Fischer, S., \& Gross, J. J. (2010). Emotion regulation and vulnerability to depression: Spontaneous versus instructed use of emotion suppression and reappraisal. Emotion, 10, 563-572.

Enns, M. W., Cox, B. J., Sareen, J., \& Freeman, P. (2001). Adaptive and maladaptive perfectionism in medical students: A longitudinal investigation. Medical Education, 35, $1034-1042$.

Feather, N. T. (1966). Effects of prior success and failure on expectations of success and subsequent performance. Journal of Personality and Social Psychology, 3, 287-298.

Flett, G. L., \& Hewitt, P. L. (2002). Perfectionism and maladjustment: An overview of theoretical, definitional, and treatment issues. In G. L. Flett \& P. L. Hewitt (Eds.), Perfectionism: Theory, research, and treatment. (pp. 5-31). Washington, DC: American Psychological Association. 
Flett, G. L., Madorsky, D., Hewitt, P. L., \& Heisel, M. J. (2002). Perfectionism cognitions, rumination, and psychological distress. Journal of Rational-Emotive and CognitiveBehavioral Therapy, 20, 33-47.

Flett, G. L., Mara, C. A., Hewitt, P. L., Sirois, F., \& Molnar, D. (2016). How should discrepancy be assessed in perfectionism research? A psychometric analysis and proposed refinement of the Almost Perfect Scale-Revised. Journal of Psychoeducational Assessment, 34, 718732.

Frost, R. O., Heimberg, R. G., Holt, C. S., Mattia, J. I., \& Neubauer, A. L. (1993). A comparison of two measures of perfectionism. Personality and Individual Differences, 14, 119-126.

Frost, R. O., \& Marten, P. A. (1990). Perfectionism and evaluative threat. Cognitive Therapy and Research, 14, 559-572.

Frost, R. O., Marten, P., Lahart, C., \& Rosenblate, R. (1990). The dimensions of perfectionism. Cognitive Therapy and Research, 19, 195-206.

Garnefski, N., Kraaij, V., \& Spinhoven, P. (2001). Negative life events, cognitive emotion regulation and emotional problems. Personality and Individual Differences, 30, 13111327.

Gilhooly, K. J., \& Johnson, C. E. (1978). Effects of solution word attributes on anagram difficulty: A regression analysis. Quarterly Journal of Experimental Psychology, 30, 5770.

Goldner, E. M., Cockell, S. J., \& Srikameswaran, S. (2002) Perfectionism and eating disorders. In G. L. Flett \& P. L. Hewitt (Eds.), Perfectionism: Theory, research, and treatment (pp. 319-340). Washington, DC: American Psychological Association. 
Greenberg, J., \& Pyszczynski, T. (1986). Persistent high self-focus after failure and low selffocus after success: The depressive self-focusing style. Journal of Personality and Social Psychology, 50, 1039-1044.

Gross, J. J. (1998). The emerging field of emotion regulation: An integrative review. Review of General Psychology, 2, 271-299.

Gross, J. J. (2008). Emotion and emotion regulation: Personality processes and individual differences. In O. P. John, R. W. Robins, \& L. A. Pervin (Eds.), Handbook of personality: Theory and research., 3rd ed. (pp. 701-724). New York, NY: Guilford Press.

Gross, J. J., \& John, O. P. (2003). Individual differences in two emotion regulation processes: Implications for affect, relationships, and well-being. Journal of Personality and Social Psychology, 85(2), 348-362.

Gross, J. J., Richards, J. M., \& John, O. P. (2006). Emotion Regulation in Everyday Life. In D. K. Snyder, J. Simpson, \& J. N. Hughes (Eds.), Emotion regulation in couples and families: Pathways to dysfunction and health. (pp. 13-35). Washington, DC: American Psychological Association.

Gross, J. J., \& Thompson, R. A. (2013). Emotion Regulation: Conceptual Foundations. In J. J. Gross (Ed.), Handbook of emotion regulation. (2nd ed.) (pp. 3-24). New York, NY: Guilford Press.

Hamachek, D. E. (1978). Psychodynamics of normal and neurotic perfectionism. Psychology, 15, 27-33.

Hayes, A. F. (2018). Introduction to mediation, moderation, and conditional process analysis (2nd ed.). New York, NY: The Guilford Press. 
Hayes, S. C., Strosahl, K., Wilson, K. G., Bissett, R. T., Pistorello, J., Toarmino, D., ... McCurry, S. M. (2004). Measuring Experiential Avoidance: A Preliminary Test of a Working Model. The Psychological Record, 54, 553-578.

Henry, J. D., \& Crawford, J. R. (2005). The short-form version of the Depression Anxiety Stress Scales (DASS-21): Construct validity and normative data in a large non-clinical sample. British Journal of Clinical Psychology, 44, 227-239.

Hewitt, P. L., \& Flett, G. L. (1991). Perfectionism in the self and social contexts: Conceptualization, assessment, and associations with psychopathology. Journal of Personality and Social Psychology, 60, 456-470.

Hewitt, P. L., Flett, G. L., Sherry, S. B., Habke, M., Parkin, M., Lam, R. W., McMurtry, B., Ediger, E., Fairlie, P., \& Stein, M. B. (2003). The interpersonal expression of perfection: Perfectionistic self-presentation and psychological distress. Journal of Personality and Social Psychology, 84, 1303-1325.

Hill, A. P., \& Davis, P. A. (2014). Perfectionism and emotion regulation in coaches: A test of the $2 \times 2$ model of dispositional perfectionism. Motivation and Emotion, 38, 715-726.

James, K., Verplanken, B., \& Rimes, K. A. (2015). Self-criticism as a mediator in the relationship between unhealthy perfectionism and distress. Personality and Individual Differences, 79, 123-128.

James, W. (1884). What is an emotion? Mind, 9(34), 188-205.

Just, N., \& Alloy, L. B. (1997). The response styles theory of depression: Tests and an extension of the theory. Journal of Abnormal Psychology, 106, 221-229. 
Kawamura, K. Y., \& Frost, R. O. (2004). Self-concealment as a mediator in the relationship between perfectionism and psychological distress. Cognitive Therapy and Research, 28, 183-191.

Limburg, K., Watson, H. J., Hagger, M. S., \& Egan, S. J. (2017). The relationship between perfectionism and psychopathology: A meta-analysis. Journal of Clinical Psychology, 73, $1301-1326$

Lo, A., \& Abbott, M. J. (2013). The impact of manipulating expected standards of performance for adaptive, maladaptive, and non-perfectionists. Cognitive Therapy and Research, 37, $762-778$

Macedo, A., Marques, C., Quaresma, V., Soares, M. J., Amaral, A. P., Araujo, A. I., \& Pereira, A. T. (2017). Are perfectionism cognitions and cognitive emotion regulation strategies mediators between perfectionism and psychological distress? Personality and Individual Differences, 119, 46-51.

Macedo, A., Soares, M. J., Amaral, A.P., Nogueira, V., Madeira, N., Roque, C., Marques, M., Maia, B., Bos, S., Valenta, J., \& Pereira, A. T. (2015). Repetitive negative thinking mediates the association between perfectionism and psychological distress. Personality and Individual Differences, 72, 220-224.

Malivoire, B. L., Kuo, J. R., \& Antony, M. M. (2019). An examination of emotion dysregulation in maladaptive perfectionism. Clinical Psychology Review, 71, 39-50.

Martin, L. L., \& Tesser, A. (1996). Some ruminative thoughts. Advances in social cognition, 9, $1-47$.

Mayer, J. D., \& Geher, G. (1996). Emotional intelligence and the identification of emotion. Intelligence, 22, 89-114. 
Molnar, D. S., Reker, D. L., Culp, N. A., Sadava, S. W., \& DeCourville, N. H. (2006). A mediated model of perfectionism, affect, and physical health. Journal of Research in Personality, 40, 482-500.

Moroz, M., \& Dunkley, D. M. (2019). Self-critical perfectionism, experiential avoidance, and depressive and anxious symptoms over two years: A three-wave longitudinal study. Behaviour Research and Therapy, 112, 18-27.

Nolen-Hoeksema, S. (2000). The role of rumination in depressive disorders and mixed anxiety/depressive symptoms. Journal of Abnormal Psychology, 109, 504-511.

O’Connor, D. B., O’Connor, R. C., \& Marshall, R. (2007). Perfectionism and psychological distress: Evidence of the mediating effects of rumination. European Journal of Personality, 21, 429-452.

O’Connor, R. C., Rasmussen, S., \& Hawton, K. (2010). Predicting depression, anxiety and selfharm in adolescents: The role of perfectionism and acute life stress. Behaviour Research and Therapy, 48, 52-59.

Park, H., Heppner, P. P., \& Lee, D. (2010). Maladaptive coping and self-esteem as mediators between perfectionism and psychological distress. Personality and Individual Differences, 48, 469-474.

Perrone-McGovern, K. M., Simon-Dack, S. L., Beduna, K. N., Williams, C. C., \& Esche, A. M. (2015). Emotions, cognitions, and well-being: The role of perfectionism, emotional overexcitability, and emotion regulation. Journal for the Education of the Gifted, 38, 343357. 
Price, M., \& Anderson, P. L. (2011). The impact of cognitive behavioral therapy on post event processing among those with social anxiety disorder. Behaviour Research and Therapy, 49, 132-137.

Rasmussen, S., \& Tsuang, M. (1986). Clinical characteristics and family history in DSM-III obsessive-compulsive disorder. American Journal of Psychiatry, 143, 317-322.

Rice, K. G., \& Ashby, J. S. (2007). An efficient method of classifying perfectionists. Journal of Counseling Psychology, 54, 72-85.

Rice, K. G., Suh, H., \& Davis, D. E. (2018). Perfectionism and emotion regulation. In J. Stoeber (Ed.), The psychology of perfectionism: Theory, research, applications. (pp. 243-262). New York, NY: Routledge/Taylor \& Francis Group.

Rice, K. G., Vergara, D. T., \& Aldea, M. A. (2006). Cognitive-affective mediators of perfectionism and college student adjustment. Personality and Individual Differences, 40, 463-473.

Richardson, C. M., \& Rice, K. G. (2015). Self-critical perfectionism, daily stress, and disclosure of daily emotional events. Journal of counseling psychology, 62, 694.

Richardson, C. M. E., Rice, K. G., \& Devine, D. P. (2014). Perfectionism, emotion regulation, and the cortisol stress response. Journal of Counseling Psychology, 61, 110-118.

Roelofs, J., Muris, P., Huibers, M., Peeters, F., \& Arntz, A. (2006). On the measurement of rumination: A psychometric evaluation of the ruminative response scale and the rumination on sadness scale in undergraduates. Journal of Behavior Therapy and Experimental Psychiatry, 37, 299-313. 
Rudolph, S. G., Flett, G. L., \& Hewitt, P. L. (2007). Perfectionism and deficits in cognitive emotion regulation. Journal of Rational-Emotive \& Cognitive Behavior Therapy, 25, 343-357.

Santanello, A. W., \& Gardner, F. L. (2007). The role of experiential avoidance in the relationship between maladaptive perfectionism and worry. Cognitive Therapy and Research, 30, 319-332.

Scherer, A., Boecker, M., Pawelzik, M., Gauggel, S., \& Forkmann, T. (2017). Emotion suppression, not reappraisal, predicts psychotherapy outcome. Psychotherapy Research, 27, 143-153.

Senra, C., Merino, H., \& Ferreiro, F. (2017). Exploring the link between perfectionism and depressive symptoms: Contribution of rumination and defense styles. Journal of Clinical Psychology, 74, 1053-1066.

Shafran, R., Cooper, Z., \& Fairburn, C. G. (2002). Clinical perfectionism: a cognitivebehavioural analysis. Behavior Research and Therapy, 40, 773-791.

Shafran, R., \& Mansell, W., (2001). Perfectionism and psychopathology: A review of research and treatment. Clinical Psychology Review, 21, 879-906.

Sheppes, G., Scheibe, S., Suri, G., \& Gross, J. J. (2011). Emotion-regulation choice. Psychological science, 22, 1391-1396.

Sironic, A., \& Reeve, R. A. (2015). A combined analysis of the Frost Multidimensional Perfectionism Scale (FMPS), Child and Adolescent Perfectionism Scale, and Almost Perfect Scale-Revised (APS-R): Different perfectionist profiles in adolescent high school students. Psychological Assessment, 27, 1471-1483. 
Slaney, R. B., Rice, K. G., \& Ashby, J. S. (2002). A programmatic approach to measuring perfectionism: The Almost Perfect Scales. In G. L. Flett \& P. L. Hewitt (Eds.), Perfectionism: Theory, research, and treatment (pp. 63-88). Washington, DC: American Psychological Association.

Slaney, R. B., Rice, K. G., Mobley, M., Trippi, J., \& Ashby, J. S. (2001). The revised almost perfect scale. Measurement and Evaluation in Counseling and Development, 34, 130145.

Smith, M. M., Saklofske, D. H., Yan, G., \& Sherry, S. B. (2017). Does perfectionism predict depression, anxiety, stress, and life satisfaction after controlling for neuroticism? A study of Canadian and Chinese undergraduates. Journal of Individual Differences, 38, 63-70.

Spinhoven, P., Klein, N., Kennis, M., Cramer, A. O. J., Siegle, G., Cuijpers, P., ... Bockting, C. L. (2018). The effects of cognitive-behavior therapy for depression on repetitive negative thinking: A meta-analysis. Behaviour Research and Therapy, 106, 71-85.

Stoeber, J. (2015). How other-oriented perfectionism differs from self-oriented and socially prescribed perfectionism. Journal of Psychopathology and Behavioral Assessment, 36, 329-338.

Stoeber, J., \& Becker, C. (2008). Perfectionism, achievement motives, and attribution of success and failure in female soccer players. International Journal of Psychology, 43, 980-987.

Stoeber, J., Schneider, N., Hussain, R., \& Matthews, K. (2014). Perfectionism and negative affect after repeated failure. Journal of Individual Differences, 35, 87-94.

Terry-Short, L. A., Owens, R. G., Slade, P. D., \& Dewey, M. E. (1995). Positive and negative perfectionism. Personality and Individual Differences, 18, 663-668. 
Treynor, W., Gonzalez, R., \& Nolen-Hoeksema, S. (2003). Rumination reconsidered: A psychometric analysis. Cognitive Therapy and Research, 27, 247-259.

Van der Kaap-Deeder, J., Soenens, B., Boone, L., Vandenkerckhove, B., Stemgee, E., Vansteenkiste, M. (2016). Evaluative concerns perfectionism and coping with failure: Effects on rumination, avoidance, and acceptance. Personality and Individual Differences, 101, 114-119.

Vogel, D. L., Wester, S. R., \& Larson, L. M. (2007). Avoidance of counseling: Psychological factors that inhibit seeking help. Journal of Counseling \& Development, 84, 410-422.

Watson, D., Clark, L. A., \& Tellegen, A. (1988). Development and validation of the brief measures of positive and negative affect: The PANAS scale. Journal of Personality and Social Psychology, 54, 1063-1070.

Wei, M., Mallinckrodt, B., Russell, D. W., \& Abraham, W. T. (2004). Maladaptive perfectionism as a mediator and moderator between adult attachment and depressive mood. Journal of Counseling Psychology, 52, 201-212.

Weston, R., \& Gore, P. A. (2006). A brief guide to structural equation modeling. The Counseling Psychologist, 34, 719-751.

Wu, T., \& Wei, M. (2008). Perfectionism and negative mood: The mediating roles of validation from others versus self. Journal of Counseling Psychology, 55, 276-288. 


\section{THORNDIKE INTELLIGENCE MEASURE ${ }^{\star}$}

Verbal Intelligence Test

INSTRUCTIONS: The following prompts include anagrams. These are words in which the letters have been rearranged. Results on the Thorndike Intelligence Test are a valid measure of intelligence. Rearrange the letters below to create words. Write your answers in the solution column.

Anagram Solution

1. HTMON

2. IUNYT

3. TELSY

4. NOEHY

5. ONECI

6. ECRVO

7. CIAHR

8. HAENY

9. ANBIT

10. MACPR

11. FTLRI

12. LAKPN

13. YAORV

14. CHITP

15. KTENO

16. LTIGN

17. UPNLO

18. ADEBL

19. GBRUY

20. DATGI

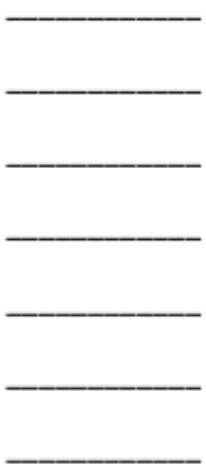

How did you do?

(within 10 minute time linit)

20 H.......... Excellent

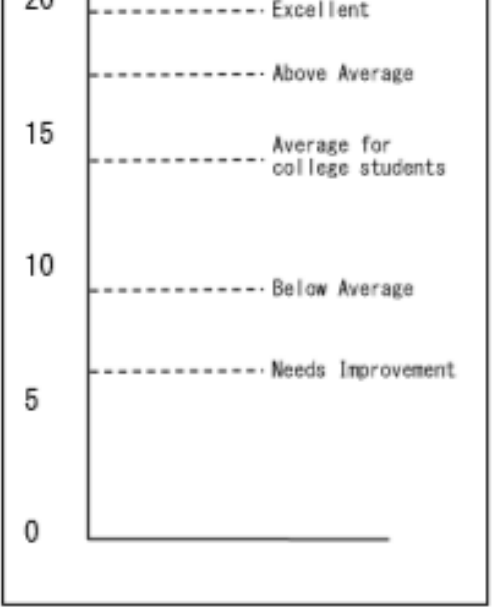

- 2019 Thorndike Psycholozical Group 


\section{THORNDIKE INTELLIGENCE MEASURE}

Verbal Intelligence Test

INSTRUCTIONS: The following prompts include anagrams. These are words in which the letters have been rearranged. Results on the Thorndike Intelligence Test are a valid measure of intelligence. Rearrange the letters below to create words. Write your answers in the solution column.

\section{$\underline{\text { Anagram } \quad \text { Solution }}$}

1. HTMON

MONTH

2. IUNYT

UNITY

3. TELSY

STYLE

4. NOEHY

HONEY

5. ONECI

NONE

6. ECRVO

COVER

7. CIAHR

CHAIR

8. HAENY

HYENA

9. ANBIT

NONE

10. MACPR

CRAMP

11. FTLRI

FLIRT

12. LAKPN

PLANK

13. YAORV

OVARY

14. CHITP

PITCH

15. KTENO

TOKEN

16. LTIGN

GLINT

17. UPNL0

NONE

18. ADEBL

BLADE

19. GBRUY

RUGBY

20. DATGI

NONE

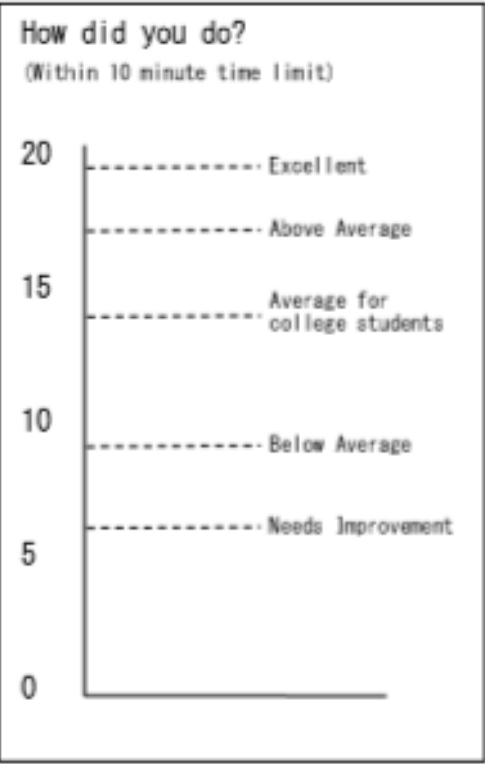




\section{THORNDIKE INTELLIGENCE MEASURE \\ Verbal Intelligence Test \\ INSTRUCTIONS: The following prompts include anagrams. These are words in which the letters have been rearranged. Results on the Thorndike Intelligence Test are a valid measure of intelligence.}

Rearrange the letters below to create words. Write your answers in the solution column.

Anagram Solution

1. CIAHR

2. ONAPR

3. EUNDC

4. ADEBL

5. HAENY

6. YQERU

7. SRIVU

8. LCKUP

9. NEIBR

10. ACNFY

11. TELSY

12. HTHON

13. TRHIM

14. EMCYR

15. LTIGN

16. JEGUD

17. BOTIR

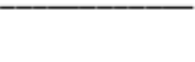

18. CNIHF
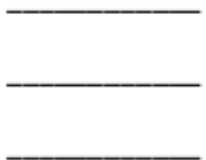

19. NIRDK

20. IJNOT

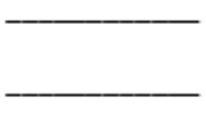

How did you do?

Within 10 ninute time l init)

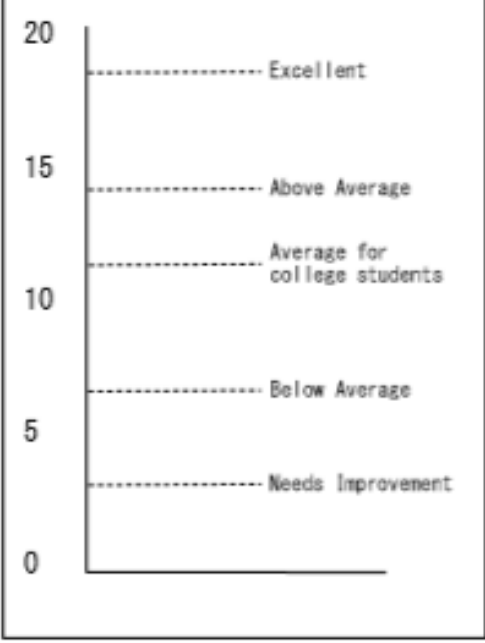




\section{THORNDIKE INTELLIGENCE MEASURE ${ }^{\mathrm{s}}$}

Verbal Intelligence Test

INSTRUCTIONS: The following prompts include anagrams. These are words in which the letters have been rearranged. Results on the Thorndike Intelligence Test are a valid measure of intelligence.

Rearrange the letters below to create words. Write your answers in the solution column.

\section{$\underline{\text { Anagram }}$}

1. CIAHR

2. ONAPR

3. EUNDC

4. ADEBL

5. HAENY

6. YQERU

7. SRIVU

8. LCKUP

9. NEIBR

10. ACNFY

11. TELSY

12. HTMON

13. TRHIM

14. EMCYR

15. LTIGN

16. JEGUD

17. BOTIR

18. CNIHF

19. NIRDK

20. IJNOT

Solution

CHAIR

APRON

DUNCE

BLADE

HYENA

QUERY

VIRUS

PLUCK

BRINE

FANCY

STYLE

MONTH

MIRTH

MERCY

GLINT

JUDGE

ORBIT

$\mathrm{FINCH}$

DRINK

JOINT

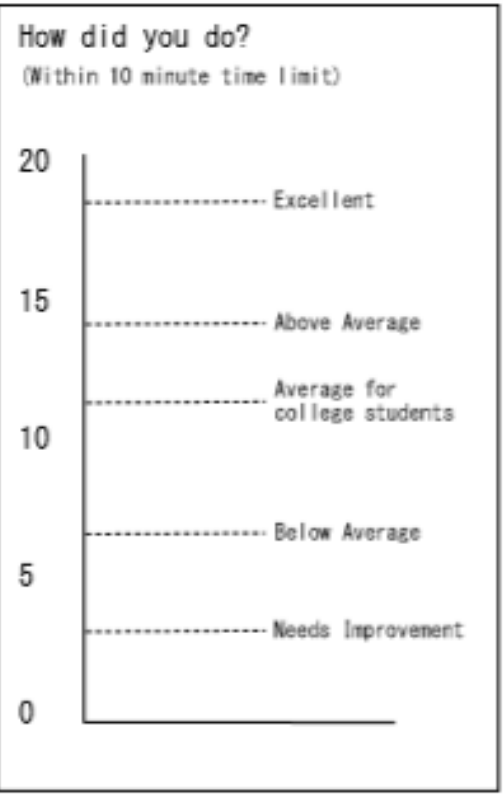




\section{APPENDIX C: INFORMED CONSENT Participant Consent Form}

You are being asked to participate in a research study conducted by Jennifer Woodrum, Graduate Student in Clinical-Counseling Psychology, and Dr. Jeffrey Kahn, Professor in the Department of Psychology at Illinois State University. The purpose of this study is to examine aspects of people's personalities, emotions, and well-being.

\section{Why are you being asked?}

You have been asked to participate because you are a student taking a psychology course in which credit is offered for research participation. Your participation in this study is voluntary. You will not be penalized if you choose to skip parts of the study, not participate, or withdraw from the study at any time.

\section{What would you do?}

If you choose to participate in this study, you will complete various questionnaires meant to gather information. You will complete a short task, followed by additional questionnaires in which you will report on your experiences. In total, your involvement in this study will last approximately 45 minutes.

\section{Are any risks expected?}

There is a small risk that you will come across a question or answer choice that you find unpleasant, upsetting, or otherwise objectionable. For example, some survey questions ask about negative feelings or emotions. Additionally, there is a small risk that you may experience subjective distress while completing one of the tasks in this study. To reduce these risks, we remind you that you do not have to answer any questions you feel uncomfortable answering.

\section{Will your information be protected?}


We will use all reasonable efforts to keep any provided personal information confidential. All questionnaires will be given codes and stored separately from any names or other direct identification of participants. Research information will be kept in locked files or on passwordprotected computers at all times. Only research personnel will have access to these files and questionnaires. Information that may identify you or potentially lead to re-identification will not be released to individuals that are not on the research team. If research results are disseminated, any information that could identify you will be removed. However, when required by law or university policy, identifying information (including your signed consent form) may be seen or copied by authorized individuals.

\section{Could your responses be used for other research?}

We will not use any identifiable information from you in future research, but your deidentified information could be used for future research without additional consent from you.

\section{Will you receive anything for participating?}

By taking part in this research study, you will be offered 1.5 points of credit through the Sona system.

\section{Who will benefit from this study?}

A benefit to you for participating is that after completing these measures and tasks, you will have an opportunity to learn more about this research, which can help you in your psychology course. By completing the questionnaires, you may also learn more about yourself. Additionally, this research may benefit society by helping us learn more about people's personalities and emotions.

\section{Whom do you contact if you have any questions?}


If you have any questions about the research or wish to withdraw from the study, contact

Jennifer Woodrum at jljaroc@ilstu.edu or Dr. Jeffrey Kahn at jhkahn@ilstu.edu or (309) 4387939.

If you have any questions about your rights as a participant, or if you feel you have been placed at risk, contact the Illinois State University Research Ethics \& Compliance Office at (309) 4385527 or IRB@ilstu.edu.

\section{Documentation of Consent}

Sign below if you are 18 or older and willing to participate in this study.

Signature

Date

You will be given a copy of this form for your records. 


\section{APPENDIX D: DEBRIEFING}

\section{DEBRIEFING}

The purpose of the tasks you just completed was to learn more about people with certain traits of perfectionism. The first questionnaires that you filled out measure aspects of personality and typical ways that you experience emotions and distress. After completing the Thorndike

Intelligence Measure, the other questionnaires that you filled out measure how you responded to a potentially distressing situation.

The purpose of doing this study is to find out how people with traits of perfectionism regulate their emotions and experience distress. To do this, we fabricated a stress-inducing situation for some participants. The Thorndike Intelligence Measure is not a measure of intelligence. The test was created for this study and the average scores that you were told were made-up.

The difficulty of the anagrams was also manipulated for this study. Some participants received a test with anagrams that were very easy. Other participants received a test with anagrams that were either extremely difficult or impossible to solve. This manipulation was created to induce an experience of acute distress.

Research in this area has implications for our understanding of perfectionism. This study can give us insight into how perfectionism affects emotion regulation and distress, which can provide us with information for treatment. Your participation has been very helpful to us, and we thank you for participating.

If you have any questions or experienced any problems with the study you can talk with Dr. Jeffrey Kahn (jhkahn@ilstu.edu, 309-438-7939). We understand that some participants might have experienced some distress as a result of participating in this study. If this has been the 
case, please consider making an appointment to meet with someone from Student Counseling Services by calling (309) 438-3655 or stopping by room 320 of the Student Services Building.

In this study it is critical that future participants do not know anything about the study until after they have participated in it. This is necessary in order to prevent people from responding in a biased manner. Therefore, it is extremely important that you keep information about your experience today to yourself. We hope that you will respect the integrity of this research study by keeping the details of this study private.

\section{SECOND CONSENT FORM}

You received a version of the Thorndike Intelligence Measure that was either very difficult or less difficult. You were told the average number of anagrams that college students can complete, but we made this information up. Some participants were given this difficult measure so we could see how perfectionists respond to distressing situations in the moment.

We are aware that the anagram test may have had an impact on your emotion regulation and experiences of distress. Some participants may wish to reconsider whether we are able to use their data in our analysis. We therefore would like to ask your permission to use your data in our study. Remember, no identifying information exists on any of the research questionnaires, and your name cannot be associated with your data. If you choose not to give us permission, your questionnaires will be discarded.

If you agree to let us use your questionnaires in our research study, please sign below: 


\section{APPENDIX E: STRATEGIES QUESTIONNAIRE}

We would like to ask you some questions about your emotional experiences while completing the Thorndike Intelligence Measure. The questions below involve three distinct aspects of your emotional life. One is your emotional experience, or what you feel like inside. The second is your emotional expression, or how you show your emotions in the way you talk, gesture, or

behave. The third has to do with ways that you think or feel. For each item, please answer using the following scale:

1 strongly disagree

7 strongly agree

1. When I was feeling negative emotions, I made sure not to express them.

2. If I started to get upset or frustrated, I changed the way I thought about my experience.

3. This task made me feel good about myself.

4. While doing this task, I thought about my shortcomings, failures, faults, and mistakes.

5. I tried to push aside my feelings while doing this task.

6. I made myself think about this task in a way that helped me stay calm.

7. While doing this task, I thought about how upset I was with myself.

8. I controlled my emotions by keeping them inside.

9. I really enjoyed doing this task.

10. While I was completing these anagrams, I kept wondering why I wasn't doing better.

11. The way that I thought about doing this task helped prevent me from getting too worked up.

12. I mostly felt positive emotions while completing this task. 


\section{APPENDIX F: STATE PERFECTIONISM QUESTIONNAIRE}

Answer the questions below about your experience while taking the Thorndike Intelligence Measure.

1 strongly disagree

7 strongly agree

1. I probably set higher goals for myself than most people on this task

2. I felt like if I failed at this task, I would be a failure as a person

3. I worried about not measuring up to my own expectations on this task

4. I had a lot of doubts about my performance on this task

5. It was important to me that I was thoroughly competent on this task

6. Even though I did this task very carefully, I often felt like it was not quite right

7. I felt disappointed after completing this task because I knew I could have done better

8. I felt upset if I made a mistake on this task 